Research Article

\title{
Common Expression Quantitative Trait Loci Shared by Histone Genes
}

\author{
Hanseol Kim, Yujin Suh, and Chaeyoung Lee \\ Department of Bioinformatics and Life Science, Soongsil University, Seoul, Republic of Korea \\ Correspondence should be addressed to Chaeyoung Lee; clee@ssu.ac.kr
}

Received 29 May 2017; Revised 26 July 2017; Accepted 2 August 2017; Published 27 August 2017

Academic Editor: Marco Gerdol

Copyright (c) 2017 Hanseol Kim et al. This is an open access article distributed under the Creative Commons Attribution License, which permits unrestricted use, distribution, and reproduction in any medium, provided the original work is properly cited.

\begin{abstract}
A genome-wide association study (GWAS) was conducted to examine expression quantitative trait loci (eQTLs) for histone genes. We examined common eQTLs for multiple histone genes in 373 European lymphoblastoid cell lines (LCLs). A linear regression model was employed to identify single-nucleotide polymorphisms (SNPs) associated with expression of the histone genes, and the number of eQTLs was determined by linkage disequilibrium analysis. Additional associations of the identified eQTLs with other genes were also examined. We identified 31 eQTLs for 29 histone genes through genome-wide analysis using 29 histone genes $\left(P<2.97 \times 10^{-10}\right)$. Among them, 12 eQTLs were associated with the expression of multiple histone genes. Transcriptomewide association analysis using the identified eQTLs showed their associations with additional 80 genes $\left(P<4.75 \times 10^{-6}\right)$. In particular, expression of RPPH1, SCARNA2, and SCARNA7 genes was associated with 26, 25, and 23 eQTLs, respectively. This study suggests that histone genes shared 12 common eQTLs that might regulate cell cycle-dependent transcription of histone and other genes. Further investigations are needed to elucidate the transcriptional mechanisms of these genes.
\end{abstract}

\section{Introduction}

Histone mRNA transcripts and proteins are important for packing DNA into chromatin and are thus tightly regulated in most human cells [1]. In humans, the genes encoding histones are gathered on chromosomes 1 and 6 . It has been suspected that the clustered structure of genes can provide a manageable unit for coordinating transcription [1]. Recently, genome-wide chromatin interaction analysis with paired-end-tag sequencing (ChIA-PET) has shown that some histone genes can share promoters [2].

While many efforts have been made to understand the mechanisms for the transcription of histone genes, they have not yet been well defined. Nuclear protein of the ataxia-telangiectasia-mutated locus (NPAT), which promotes the transcription of histone genes, is located near the Cajal body [1]. Clusters of histone genes are also located near the Cajal body [3]. The positions of histone gene clusters near the Cajal body have been observed between the restriction point (R-point) and the G1/S transition (S-point) during the cell cycle [4]. The objective of this study was to select simultaneously expressed histone genes, identify their expression quantitative trait loci (eQTLs), and examine the functions of those eQTLs.

\section{Material and Methods}

2.1. Subjects and Data. The subjects of this study were 373 Europeans including 95 Finnish in Finland, 94 British in England and Scotland, 93 Tuscans from Italy, and 91 Utahn residents with Northern and Western European ancestry from the CEPH collection. Their genotypic data were derived from the phase 1 dataset produced by the 1000 Genomes Project [5] (http://www.internationalgenome.org/). This study utilized genotypic data at 5,796,145 SNPs after filtering out the SNPs with minor allele frequency $<0.05$, with missing rate $>0.05$, or in Hardy-Weinberg disequilibrium with $P<0.001$.

Transcriptional data on 10,518 human genes were obtained in lymphoblastoid cells of the subjects by the Geuvadis RNA sequencing project (http://www.geuvadis.org/ web/geuvadis/rnaseq-project). The unit used for the mRNA expression level was reads per kilobase per million mapped 


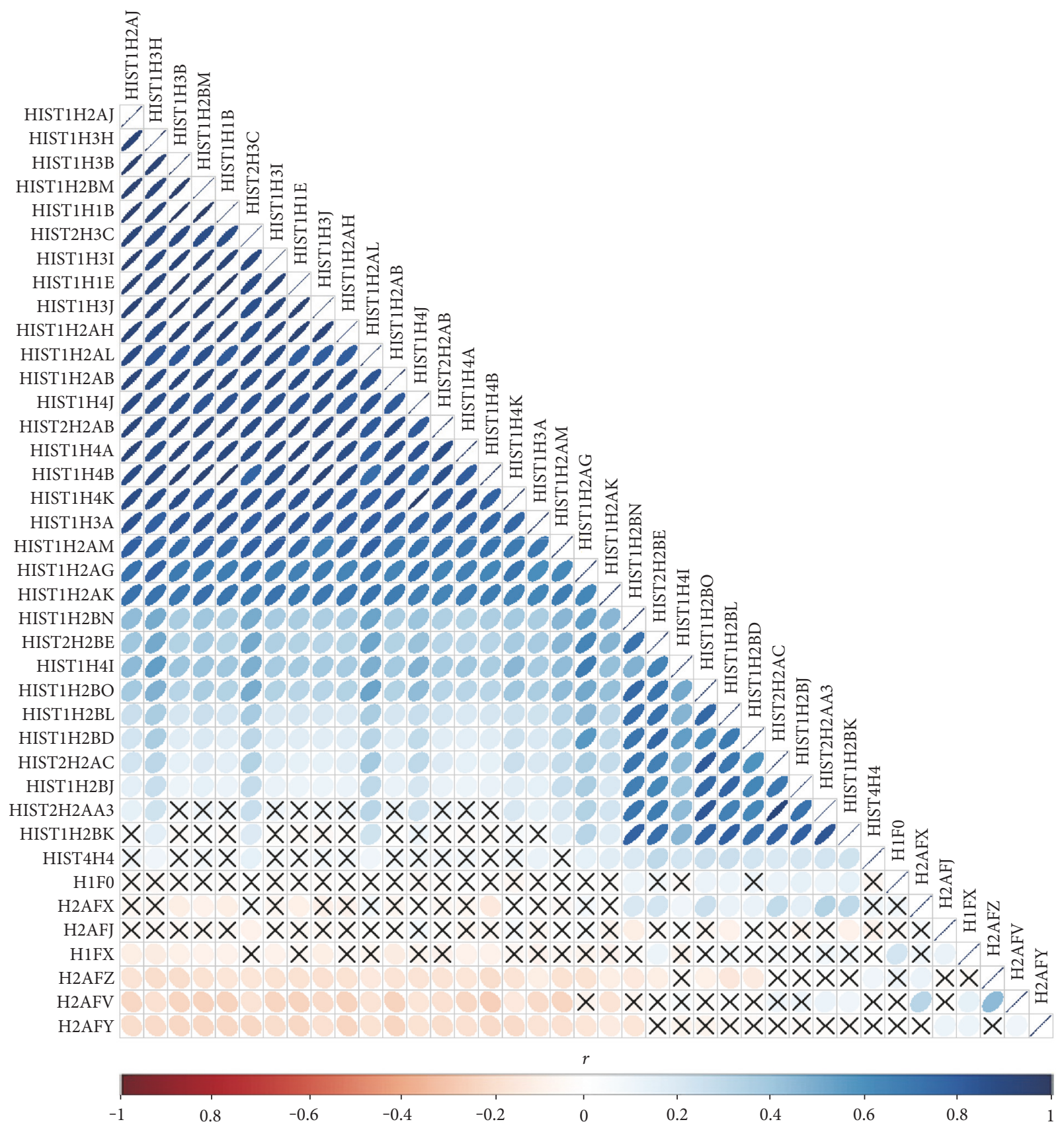

Figure 1: Pearson's correlation $(r)$ between expressions of histone genes using the ellipse visualization method. The correlation estimate without significance $(P>0.05)$ is marked with "X."

reads (RPKM). Outliers were removed based on sample similarity, which was estimated by the Spearman rank correlation between RPKMs and the exon counts of the samples [6]. Sample swaps or contaminated samples were excluded based on allele-specific expression analysis [6]. For details on the quality control process, see t Hoen et al. [7].

2.2. Statistical Methods. We selected histone genes that were expressed simultaneously. Pairwise gene expression relationships were estimated using Pearson's correlation coefficient $(r)$. The significance of the correlation was determined by $P<0.05$.
We investigated genome-wide associations of the expression of the selected histone genes. A regression model was employed to identify SNPs associated with expressions of histone genes using PLINK [8]. The Bonferroni correction was applied as a multiple testing, and the significance was determined by $P<2.97 \times 10^{-10}$.

Linkage disequilibrium (LD) between the identified SNPs was estimated using the HaploView program [9]. The LD block was determined according to the $95 \%$ confidence interval of the $D^{\prime}$ value for pairwise LD between the nucleotide variants with minor allele frequency $>0.05[10]$. 
TABLE 1: Genome-wide associations of SNPs with expression of histone genes.

\begin{tabular}{|c|c|c|c|c|c|c|c|c|c|}
\hline $\mathrm{SNP}^{\mathrm{a}}$ & Gene & Effect $^{\text {b }}$ & Location $^{\mathrm{c}}$ & $\mathrm{A} 1^{\mathrm{d}}$ & $\mathrm{A} 2^{\mathrm{d}}$ & MAF & Genetic associations & Beta & $P$ \\
\hline rs10919229 & SELE (2Kb upstream) & & $1: 169735986$ & $\mathrm{~T}$ & A & 0.05 & HIST1H4B & 1.035 & $2.51 E-10$ \\
\hline rs191508159 & Intergenic & & 2:35957035 & $\mathrm{T}$ & $\mathrm{C}$ & 0.06 & HIST1H4A & 0.466 & $3.41 E-11$ \\
\hline rs79103588 & Intergenic & & 2:48891009 & A & $\mathrm{T}$ & 0.05 & HIST1H4B & 1.045 & $1.02 E-10$ \\
\hline rs10490124 & Intergenic & & $2: 48897335$ & G & A & 0.06 & HIST1H4B & 1.023 & $1.65 E-10$ \\
\hline rs7562208 & Intergenic & & 2:48898231 & $\mathrm{C}$ & $\mathrm{T}$ & 0.06 & HIST1H4B & 1.023 & $1.65 E-10$ \\
\hline rs12468670 & Intergenic & & 2:48902054 & $\mathrm{T}$ & G & 0.06 & HIST1H4B & 1.023 & $1.65 E-10$ \\
\hline rs7563070 & Intergenic & & 2:48902202 & $\mathrm{T}$ & $\mathrm{C}$ & 0.06 & HIST1H4B & 1.023 & $1.65 E-10$ \\
\hline rs7563889 & Intergenic & CTCF & 2:48902751 & $\mathrm{T}$ & G & 0.06 & HIST1H4B & 1.023 & $1.65 E-10$ \\
\hline rs115071514 & Intergenic & & 2:48902928 & $\mathrm{T}$ & $\mathrm{C}$ & 0.06 & HIST1H4B & 1.023 & $1.65 E-10$ \\
\hline rs7600883 & Intergenic & & 2:48905331 & G & A & 0.06 & HIST1H4B & 1.023 & $1.65 E-10$ \\
\hline rs10170423 & Intergenic & & 2:48906784 & G & A & 0.06 & HIST1H4B & 1.023 & $1.65 E-10$ \\
\hline rs10208418 & Intergenic & & 2:48912244 & A & $\mathrm{T}$ & 0.05 & HIST1H4B & 1.079 & $3.82 E-11$ \\
\hline rs10208981 & Intergenic & & 2:48912890 & G & $\mathrm{T}$ & 0.05 & HIST1H4B & 1.049 & $8.60 E-11$ \\
\hline rs79335804 & Intergenic & & 2:48915424 & $\mathrm{T}$ & $\mathrm{C}$ & 0.05 & HIST1H4B & 1.079 & $3.82 E-11$ \\
\hline rs13430743 & Intergenic & & 2:48916396 & $\mathrm{T}$ & G & 0.05 & HIST1H4B & 1.079 & $3.82 E-11$ \\
\hline rs13429699 & Intergenic & & 2:48921530 & G & $\mathrm{C}$ & 0.05 & HIST1H4B & 1.049 & $8.60 E-11$ \\
\hline rs13432631 & Intergenic & & 2:48921948 & A & $\mathrm{C}$ & 0.05 & HIST1H4B & 1.108 & $1.78 E-11$ \\
\hline rs13385591 & Intergenic & & 2:48923227 & $\mathrm{T}$ & $\mathrm{C}$ & 0.05 & HIST1H4B & 1.079 & $3.82 E-11$ \\
\hline rs7602404 & Intergenic & & 2:48923414 & A & G & 0.05 & HIST1H4B & 1.049 & $8.60 E-11$ \\
\hline rs 142402253 & Intergenic & & 2:48924921 & A & G & 0.05 & HIST1H4B & 1.049 & $8.60 E-11$ \\
\hline \multirow{6}{*}{ rs189340111 } & \multirow{6}{*}{ Intergenic } & & \multirow{6}{*}{$2: 68450091$} & \multirow{6}{*}{ G } & \multirow{6}{*}{ A } & \multirow{6}{*}{0.06} & HIST1H4B & 0.995 & $2.99 E-12$ \\
\hline & & & & & & & HIST1H1B & 2.319 & $2.91 E-11$ \\
\hline & & & & & & & HIST1H1E & 2.044 & $6.90 E-11$ \\
\hline & & & & & & & HIST1H3J & 1.044 & $8.17 E-11$ \\
\hline & & & & & & & HIST1H2AH & 0.745 & $2.34 E-10$ \\
\hline & & & & & & & HIST1H2BM & 1.027 & $2.58 E-10$ \\
\hline \multirow{4}{*}{ rs75372391 } & \multirow{4}{*}{ LRP1B (intron 2) } & & \multirow{4}{*}{$2: 141415941$} & \multirow{4}{*}{$\mathrm{G}$} & \multirow{4}{*}{ A } & \multirow{4}{*}{0.05} & HIST1H2BM & 1.268 & $6.19 E-12$ \\
\hline & & & & & & & HIST1H4B & 1.102 & $1.39 E-11$ \\
\hline & & & & & & & HIST1H1B & 2.645 & $2.92 E-11$ \\
\hline & & & & & & & HIST1H3B & 2.016 & $2.00 E-10$ \\
\hline \multirow{4}{*}{ rs79503131 } & \multirow{4}{*}{ LRP1B (intron 2) } & & \multirow{4}{*}{$2: 141417401$} & \multirow{4}{*}{$\mathrm{T}$} & \multirow{4}{*}{$\mathrm{C}$} & \multirow{4}{*}{0.05} & HIST1H2BM & 1.268 & $6.19 E-12$ \\
\hline & & & & & & & HIST1H4B & 1.102 & $1.39 E-11$ \\
\hline & & & & & & & HIST1H1B & 2.645 & $2.92 E-11$ \\
\hline & & & & & & & HIST1H3B & 2.016 & $2.00 E-10$ \\
\hline \multirow{3}{*}{ rs76908315 } & \multirow{3}{*}{ LRP1B (intron 2) } & & \multirow{3}{*}{$2: 141428575$} & & & & HIST1H2BM & 1.246 & $9.09 E-12$ \\
\hline & & & & G & A & 0.05 & HIST1H4B & 1.080 & $2.15 E-11$ \\
\hline & & & & & & & HIST1H1B & 2.590 & $4.75 E-11$ \\
\hline & & & & & & & HIST1H2BM & 1.246 & $9.09 E-12$ \\
\hline rs75358328 & LRP1B (intron 2) & & $2: 141435763$ & $\mathrm{C}$ & $\mathrm{T}$ & 0.05 & HIST1H4B & 1.080 & $2.15 E-11$ \\
\hline & & & & & & & HIST1H1B & 2.590 & $4.75 E-11$ \\
\hline & & & & & & & HIST1H2BM & 1.246 & $9.09 E-12$ \\
\hline rs79931187 & LRP1B (intron 2) & & $2: 141440679$ & $\mathrm{C}$ & $\mathrm{T}$ & 0.05 & HIST1H4B & 1.080 & $2.15 E-11$ \\
\hline & & & & & & & HIST1H1B & 2.590 & $4.75 E-11$ \\
\hline & & & & & & & HIST1H2BM & 1.246 & $9.09 E-12$ \\
\hline rs114273249 & LRP1B (intron 2) & & 2:141441139 & $\mathrm{T}$ & $\mathrm{C}$ & 0.05 & HIST1H4B & 1.080 & $2.15 E-11$ \\
\hline & & & & & & & HIST1H1B & 2.590 & $4.75 E-11$ \\
\hline
\end{tabular}


TABle 1: Continued.

\begin{tabular}{|c|c|c|c|c|c|c|c|c|c|}
\hline $\mathrm{SNP}^{\mathrm{a}}$ & Gene & Effect $^{\mathrm{b}}$ & Location $^{\mathrm{c}}$ & $\mathrm{A} 1^{\mathrm{d}}$ & $\mathrm{A} 2^{\mathrm{d}}$ & MAF & Genetic associations & Beta & $P$ \\
\hline \multirow{3}{*}{ rs74871811 } & \multirow{3}{*}{ LRP1B (intron 2) } & & \multirow{3}{*}{$2: 141441981$} & \multirow{3}{*}{$\mathrm{T}$} & \multirow{3}{*}{$\mathrm{A}$} & \multirow{3}{*}{0.05} & HIST1H2BM & 1.246 & $9.09 E-12$ \\
\hline & & & & & & & HIST1H4B & 1.080 & $2.15 E-11$ \\
\hline & & & & & & & HIST1H1B & 2.590 & $4.75 E-11$ \\
\hline \multirow{3}{*}{ rs114288750 } & \multirow{3}{*}{ LRP1B (intron 2) } & & \multirow{3}{*}{$2: 141442588$} & \multirow{3}{*}{ A } & \multirow{3}{*}{$\mathrm{C}$} & \multirow{3}{*}{0.05} & HIST1H2BM & 1.246 & $9.09 E-12$ \\
\hline & & & & & & & HIST1H4B & 1.080 & $2.15 E-11$ \\
\hline & & & & & & & HIST1H1B & 2.590 & $4.75 E-11$ \\
\hline \multirow{3}{*}{ rs137985802 } & \multirow{3}{*}{ LRP1B (intron 2) } & & \multirow{3}{*}{$2: 141442673$} & \multirow{3}{*}{$\mathrm{T}$} & \multirow{3}{*}{$\mathrm{C}$} & \multirow{3}{*}{0.05} & HIST1H2BM & 1.246 & $9.09 E-12$ \\
\hline & & & & & & & HIST1H4B & 1.080 & $2.15 E-11$ \\
\hline & & & & & & & HIST1H1B & 2.590 & $4.75 E-11$ \\
\hline \multirow{3}{*}{ rs114372180 } & \multirow{3}{*}{ LRP1B (intron 2) } & & & & & & HIST1H2BM & 1.246 & $9.09 E-12$ \\
\hline & & & $2: 141442782$ & A & G & 0.05 & HIST1H4B & 1.080 & $2.15 E-11$ \\
\hline & & & & & & & HIST1H1B & 2.590 & $4.75 E-11$ \\
\hline & & & & & & & HIST1H2BM & 1.236 & $1.33 E-11$ \\
\hline rs75148923 & LRP1B (intron 2) & & $2: 141454893$ & $\mathrm{~T}$ & $\mathrm{C}$ & 0.05 & HIST1H4B & 1.080 & $2.15 E-11$ \\
\hline & & & & & & & HIST1H1B & 2.588 & $4.96 E-11$ \\
\hline rs72853591 & LRP1B (intron 2) & $\mathrm{OC}$ & $2: 141557777$ & $\mathrm{C}$ & $\mathrm{T}$ & 0.06 & HIST1H4B & 1.015 & $9.68 E-11$ \\
\hline & & & & & & & HIST1H1B & 2.975 & $1.62 E-14$ \\
\hline & & & & & & & HIST1H2BM & 1.369 & $3.12 E-14$ \\
\hline & & & & & & & HIST1H3B & 2.277 & $1.75 E-13$ \\
\hline & & & & & & & HIST1H4B & 1.154 & $4.90 E-13$ \\
\hline & & & & & & & HIST1H2AB & 0.310 & $8.94 E-13$ \\
\hline & & & & & & & HIST1H3J & 1.273 & $1.28 E-12$ \\
\hline & & & & & & & HIST1H1E & 2.439 & $3.33 E-12$ \\
\hline & & & & & & & HIST1H2AJ & 1.711 & $4.01 E-12$ \\
\hline rs73963540 & LRP1B (intron 2) & & $2: 141710041$ & $\mathrm{~T}$ & $\mathrm{C}$ & 0.05 & HIST1H2AL & 0.552 & $9.35 E-12$ \\
\hline & & & & & & & HIST2H2AB & 0.263 & $1.43 E-11$ \\
\hline & & & & & & & HIST2H3C & 0.609 & $4.10 E-11$ \\
\hline & & & & & & & HIST1H4K & 1.649 & $4.54 E-11$ \\
\hline & & & & & & & HIST1H4J & 2.204 & $4.67 E-11$ \\
\hline & & & & & & & HIST1H3H & 1.277 & $5.05 E-11$ \\
\hline & & & & & & & HIST1H3I & 0.574 & $6.66 E-11$ \\
\hline & & & & & & & HIST3H2BB & 0.268 & $6.82 E-11$ \\
\hline & & & & & & & HIST1H4A & 0.474 & $1.04 E-10$ \\
\hline & & & & & & & HIST1H1B & 2.975 & $1.62 E-14$ \\
\hline & & & & & & & HIST1H2BM & 1.369 & $3.12 E-14$ \\
\hline & & & & & & & HIST1H3B & 2.277 & $1.75 E-13$ \\
\hline rs17801458 & LRP1B (intron 2) & & $2: 141710903$ & $\mathrm{~T}$ & $\mathrm{C}$ & 0.05 & HIST1H4B & 1.154 & $4.90 E-13$ \\
\hline & & & & & & & HIST1H2AB & 0.310 & $8.94 E-13$ \\
\hline & & & & & & & HIST1H3J & 1.273 & $1.28 E-12$ \\
\hline & & & & & & & HIST1H1E & 2.439 & $3.33 E-12$ \\
\hline & & & & & & & HIST1H2AJ & 1.711 & $4.01 E-12$ \\
\hline & & & & & & & HIST1H2AL & 0.552 & $9.35 E-12$ \\
\hline & & & & & & & HIST2H2AB & 0.263 & $1.43 E-11$ \\
\hline & & & & & & & HIST2H3C & 0.609 & $4.10 E-11$ \\
\hline & & & & & & & HIST1H4K & 1.649 & $4.54 E-11$ \\
\hline & & & & & & & HIST1H4J & 2.204 & $4.67 E-11$ \\
\hline & & & & & & & HIST1H3H & 1.277 & $5.05 E-11$ \\
\hline
\end{tabular}


TABle 1: Continued.

\begin{tabular}{|c|c|c|c|c|c|c|c|c|c|}
\hline $\mathrm{SNP}^{\mathrm{a}}$ & Gene & Effect $^{\mathrm{b}}$ & Location $^{\mathrm{c}}$ & $\mathrm{A} 1^{\mathrm{d}}$ & $A 2^{\mathrm{d}}$ & MAF & Genetic associations & Beta & $P$ \\
\hline & & & & & & & HIST1H3I & 0.574 & $6.66 E-11$ \\
\hline & & & & & & & HIST3H2BB & 0.268 & $6.82 E-11$ \\
\hline & & & & & & & HIST1H4A & 0.474 & $1.04 E-10$ \\
\hline \multirow{14}{*}{ rs7570091 } & \multirow{14}{*}{ LRP1B (intron 2) } & & \multirow{14}{*}{$2: 141714131$} & \multirow{14}{*}{ G } & \multirow{14}{*}{$\mathrm{T}$} & \multirow{14}{*}{0.05} & HIST1H2BM & 1.297 & $1.36 E-13$ \\
\hline & & & & & & & HIST1H1B & 2.787 & $1.48 E-13$ \\
\hline & & & & & & & HIST1H3B & 2.132 & $1.34 E-12$ \\
\hline & & & & & & & HIST1H2AL & 0.543 & $4.65 E-12$ \\
\hline & & & & & & & HIST1H4B & 1.074 & $4.79 E-12$ \\
\hline & & & & & & & HIST1H3J & 1.201 & $5.93 E-12$ \\
\hline & & & & & & & HIST1H2AB & 0.289 & $7.28 E-12$ \\
\hline & & & & & & & HIST1H2AJ & 1.628 & $1.12 E-11$ \\
\hline & & & & & & & HIST2H2AB & 0.256 & $1.13 E-11$ \\
\hline & & & & & & & HIST3H2BB & 0.267 & $1.89 E-11$ \\
\hline & & & & & & & HIST1H1E & 2.277 & $2.38 E-11$ \\
\hline & & & & & & & $\mathrm{HIST} 2 \mathrm{H} 3 \mathrm{C}$ & 0.583 & $7.77 E-11$ \\
\hline & & & & & & & HIST1H3I & 0.545 & $1.78 E-10$ \\
\hline & & & & & & & HIST1H3H & 1.199 & $2.26 E-10$ \\
\hline \multirow{6}{*}{ rs11684178 } & \multirow{6}{*}{ LRP1B (intron 2) } & & \multirow{6}{*}{$2: 141788340$} & \multirow{6}{*}{$\mathrm{C}$} & \multirow{6}{*}{ G } & \multirow{6}{*}{0.06} & HIST1H2BM & 1.090 & $6.71 E-12$ \\
\hline & & & & & & & HIST1H1B & 2.283 & $2.56 E-11$ \\
\hline & & & & & & & HIST1H3B & 1.778 & $6.72 E-11$ \\
\hline & & & & & & & HIST2H2AB & 0.221 & $1.04 E-10$ \\
\hline & & & & & & & HIST1H3J & 1.019 & $1.10 E-10$ \\
\hline & & & & & & & HIST1H2AL & 0.455 & $1.67 E-10$ \\
\hline rs6718590 & LRP1B (intron 1) & & 2:141817609 & G & $\mathrm{C}$ & 0.07 & HIST1H2BM & 0.968 & $1.15 E-10$ \\
\hline \multirow{2}{*}{ rs111875224 } & \multirow{2}{*}{ Intergenic } & & \multirow{2}{*}{$2: 202309382$} & \multirow{2}{*}{ A } & \multirow{2}{*}{$\mathrm{C}$} & \multirow{2}{*}{0.05} & HIST1H2AM & 0.594 & $1.24 E-11$ \\
\hline & & & & & & & HIST1H4B & 1.023 & $8.51 E-11$ \\
\hline rs849583 & NRP2 (intron 7) & & 2:205737518 & A & G & 0.08 & HIST1H4A & 0.398 & $8.66 E-11$ \\
\hline rs849578 & NRP2 (intron 7) & & $2: 205738798$ & $\mathrm{~T}$ & G & 0.08 & HIST1H4A & 0.390 & $1.69 E-10$ \\
\hline rs849577 & NRP2 (intron 7) & & 2:205739705 & A & G & 0.07 & HIST1H4A & 0.407 & $4.48 E-11$ \\
\hline rs849573 & NRP2 (intron 8) & & 2:205741099 & $\mathrm{T}$ & $\mathrm{C}$ & 0.08 & HIST1H4A & 0.407 & $4.48 E-11$ \\
\hline rs849572 & NRP2 (intron 8) & & 2:205741317 & A & G & 0.08 & HIST1H4A & 0.398 & $8.98 E-11$ \\
\hline rs849567 & NRP2 (intron 9) & & $2: 205744540$ & $\mathrm{~T}$ & $\mathrm{C}$ & 0.07 & HIST1H4A & 0.403 & $7.11 E-11$ \\
\hline rs830612 & Intergenic & & $3: 71634179$ & $\mathrm{C}$ & $\mathrm{T}$ & 0.08 & HIST1H4B & 0.889 & $1.28 E-10$ \\
\hline rs150958961 & Intergenic & & 4:9006930 & $\mathrm{C}$ & A & 0.1 & HIST1H4A & 0.341 & $8.77 E-11$ \\
\hline rs148619316 & Intergenic & & 5:14080602 & $\mathrm{T}$ & $\mathrm{C}$ & 0.06 & HIST1H4B & 1.006 & $4.81 E-11$ \\
\hline rs1992368 & Intergenic & & 5:14092868 & A & G & 0.06 & HIST1H4B & 0.974 & $9.25 E-11$ \\
\hline rs55989629 & Intergenic & & 5:14093354 & $\mathrm{T}$ & $\mathrm{C}$ & 0.06 & HIST1H4B & 0.974 & $9.25 E-11$ \\
\hline rs11956277 & Intergenic & & 5:14095038 & $\mathrm{T}$ & $\mathrm{C}$ & 0.06 & HIST1H4B & 0.974 & $9.25 E-11$ \\
\hline rs66689811 & Intergenic & & 5:14095266 & $\mathrm{T}$ & $\mathrm{C}$ & 0.06 & HIST1H4B & 0.974 & $9.25 E-11$ \\
\hline rs6863067 & Intergenic & & 5:14098914 & A & $\mathrm{C}$ & 0.06 & HIST1H4B & 0.974 & $9.25 E-11$ \\
\hline rs 10041963 & Intergenic & & $5: 14099801$ & A & G & 0.06 & HIST1H4B & 0.974 & $9.25 E-11$ \\
\hline rs 10072716 & Intergenic & & $5: 14101222$ & $\mathrm{~T}$ & $\mathrm{C}$ & 0.06 & HIST1H4B & 0.974 & $9.25 E-11$ \\
\hline rs6861945 & Intergenic & & $5: 53398953$ & A & G & 0.11 & HIST1H3J & 0.780 & $2.75 E-10$ \\
\hline rs61564830 & Intergenic & & $6: 129768950$ & $\mathrm{~T}$ & $\mathrm{C}$ & 0.06 & HIST1H3J & 1.036 & $2.62 E-10$ \\
\hline rs144499291 & UTRN (intron 22) & & $6: 144471095$ & G & A & 0.06 & HIST1H4A & 0.461 & $2.88 E-10$ \\
\hline
\end{tabular}


TABle 1: Continued.

\begin{tabular}{|c|c|c|c|c|c|c|c|c|c|}
\hline $\mathrm{SNP}^{\mathrm{a}}$ & Gene & Effect $^{\mathrm{b}}$ & Location $^{\mathrm{c}}$ & $\mathrm{A} 1^{\mathrm{d}}$ & $\mathrm{A} 2^{\mathrm{d}}$ & MAF & Genetic associations & Beta & $P$ \\
\hline \multirow{2}{*}{ rs71560740 } & \multirow{2}{*}{ Intergenic } & & \multirow{2}{*}{$7: 85896218$} & \multirow{2}{*}{$\mathrm{C}$} & \multirow{2}{*}{$\mathrm{T}$} & \multirow{2}{*}{0.06} & HIST1H2AJ & 1.491 & $1.48 E-10$ \\
\hline & & & & & & & HIST1H3A & 0.228 & $2.41 E-10$ \\
\hline \multirow{12}{*}{ rs118052517 } & \multirow{12}{*}{ Intergenic } & & \multirow{12}{*}{$7: 153287269$} & \multirow{12}{*}{$\mathrm{C}$} & \multirow{12}{*}{$\mathrm{T}$} & \multirow{12}{*}{0.06} & HIST1H3J & 1.234 & $8.16 E-13$ \\
\hline & & & & & & & HIST1H4A & 0.494 & $1.89 E-12$ \\
\hline & & & & & & & HIST1H2BM & 1.222 & $2.40 E-12$ \\
\hline & & & & & & & HIST1H2AJ & 1.648 & $3.64 E-12$ \\
\hline & & & & & & & HIST1H3B & 2.052 & $6.16 E-12$ \\
\hline & & & & & & & HIST1H1B & 2.540 & $1.39 E-11$ \\
\hline & & & & & & & HIST1H1E & 2.269 & $1.78 E-11$ \\
\hline & & & & & & & HIST1H2AB & 0.270 & $1.16 E-10$ \\
\hline & & & & & & & HIST1H4K & 1.532 & $2.14 E-10$ \\
\hline & & & & & & & HIST1H4J & 2.039 & $2.62 E-10$ \\
\hline & & & & & & & HIST1H3I & 0.534 & $2.77 E-10$ \\
\hline & & & & & & & HIST1H3H & 1.180 & $2.95 E-10$ \\
\hline rs77957383 & Intergenic & & $9: 1715766$ & $\mathrm{~T}$ & $\mathrm{C}$ & 0.07 & HIST1H4B & 0.894 & $1.85 E-10$ \\
\hline rs113936625 & Intergenic & & 10:111124417 & $\mathrm{C}$ & G & 0.05 & HIST1H4B & 1.056 & $2.76 E-10$ \\
\hline \multirow{4}{*}{ rs75201173 } & \multirow{4}{*}{ Intergenic } & \multirow{4}{*}{ CTCF } & \multirow{4}{*}{$11: 1062030$} & \multirow{4}{*}{$\mathrm{C}$} & \multirow{4}{*}{ A } & \multirow{4}{*}{0.07} & HIST1H2BM & 1.092 & $1.45 E-11$ \\
\hline & & & & & & & HIST2H3C & 0.529 & $1.22 E-10$ \\
\hline & & & & & & & HIST1H1B & 2.227 & $1.75 E-10$ \\
\hline & & & & & & & HIST1H3B & 1.766 & $1.94 E-10$ \\
\hline \multirow{4}{*}{ rs11245920 } & & & & & & & HIST1H2BM & 1.092 & $1.45 E-11$ \\
\hline & Interrenis & CTCF & $11 \cdot 1062510$ & $C$ & $\mathrm{~T}$ & 007 & HIST2H3C & 0.529 & $1.22 E-10$ \\
\hline & intergenic & CICr & 11.1002519 & C & 1 & 0.07 & HIST1H1B & 2.227 & $1.75 E-10$ \\
\hline & & & & & & & HIST1H3B & 1.766 & $1.94 E-10$ \\
\hline & & & & & & & HIST1H2BM & 1.092 & $1.45 E-11$ \\
\hline rc111452409 & Intercenir & & $11 \cdot 1064920$ & $G_{1}$ & $A$ & 007 & $\mathrm{HIST} 2 \mathrm{H} 3 \mathrm{C}$ & 0.529 & $1.22 E-10$ \\
\hline rs111452409 & intergenic & & 11:1004929 & G & A & 0.07 & HIST1H1B & 2.227 & $1.75 E-10$ \\
\hline & & & & & & & HIST1H3B & 1.766 & $1.94 E-10$ \\
\hline rc1 13650777 & MUC2 (intron 2) & & $11 \cdot 1088140$ & $A$ & $\mathrm{C}_{\mathrm{N}}$ & 007 & HIST1H2BM & 1.101 & $2.89 E-11$ \\
\hline 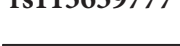 & 1910 & & 11.1000147 & $\Lambda$ & S & 0.07 & HIST1H4B & 0.951 & $8.17 E-11$ \\
\hline & & & & & & & HIST1H2BM & 1.262 & $1.56 E-12$ \\
\hline & & & & & & & HIST1H4B & 1.077 & $9.13 E-12$ \\
\hline & & & & & & & HIST1H1B & 2.566 & $2.84 E-11$ \\
\hline rs184239416 & MUC2 (intron 2) & & 11:1088154 & G & A & 0.06 & HIST1H3J & 1.174 & $3.88 E-11$ \\
\hline & & & & & & & HIST1H3B & 1.979 & $1.17 E-10$ \\
\hline & & & & & & & HIST1H1E & 2.206 & $2.06 E-10$ \\
\hline & & & & & & & HIST3H2BB & 0.257 & $2.37 E-10$ \\
\hline & & & & & & & HIST1H2BM & 1.068 & $4.19 E-11$ \\
\hline rs41392245 & MUC2 (intron 2) & & $11 \cdot 1089095$ & $\mathrm{~T}$ & $C$ & 007 & $\mathrm{HIST} 2 \mathrm{H} 3 \mathrm{C}$ & 0.528 & $1.34 E-10$ \\
\hline 1041072240 & $1010-2(111100112)$ & & 11.1007070 & 1 & C & 0.07 & HIST1H3B & 1.761 & $2.23 E-10$ \\
\hline & & & & & & & HIST1H1B & 2.201 & $2.90 E-10$ \\
\hline & & & & & & & HIST1H2AJ & 1.711 & $2.53 E-13$ \\
\hline & & & & & & & HIST1H1E & 2.374 & $9.71 E-13$ \\
\hline rs11033879 & MMP26 (intron 1) & & $11 \cdot 4786997$ & $G$ & A & 0.05 & HIST1H3B & 2.102 & $9.80 E-13$ \\
\hline $15110030 / 9$ & 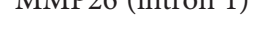 & & 11.4/0099/ & U & $\mathrm{A}$ & 0.03 & HIST2H2AB & 0.263 & $1.12 E-12$ \\
\hline & & & & & & & HIST1H1B & 2.635 & $1.18 E-12$ \\
\hline & & & & & & & HIST1H4A & 0.490 & $1.62 E-12$ \\
\hline
\end{tabular}


TABLE 1: Continued.

\begin{tabular}{|c|c|c|c|c|c|c|c|c|c|}
\hline $\mathrm{SNP}^{\mathrm{a}}$ & Gene & Effect $^{\mathrm{b}}$ & Location $^{\mathrm{c}}$ & $\mathrm{A} 1^{\mathrm{d}}$ & $\mathrm{A} 2^{\mathrm{d}}$ & MAF & Genetic associations & Beta & $P$ \\
\hline & & & & & & & HIST1H2BM & 1.195 & $4.53 E-12$ \\
\hline & & & & & & & HIST2H3C & 0.594 & $1.15 E-11$ \\
\hline & & & & & & & HIST1H3J & 1.148 & $2.08 E-11$ \\
\hline & & & & & & & HIST1H4K & 1.593 & $2.16 E-11$ \\
\hline & & & & & & & HIST1H2AB & 0.275 & $3.14 E-11$ \\
\hline & & & & & & & HIST1H4J & 2.095 & $4.85 E-11$ \\
\hline & & & & & & & HIST1H3I & 0.544 & $7.46 E-11$ \\
\hline & & & & & & & HIST1H3H & 1.190 & $1.28 E-10$ \\
\hline & & & & & & & HIST1H2AH & 0.793 & $2.70 E-10$ \\
\hline rs80143982 & Intergenic & & 11:110990257 & A & G & 0.05 & HIST1H4A & 0.482 & $2.65 E-10$ \\
\hline rs75349221 & Intergenic & & $14: 44176974$ & $\mathrm{~T}$ & A & 0.05 & HIST1H4A & 0.471 & $2.75 E-10$ \\
\hline rs72846811 & RBFOX3 (intron 3) & & $17: 79231229$ & A & G & 0.06 & HIST1H4A & 0.489 & $1.90 E-11$ \\
\hline rs183898825 & TAF4B (intron 9) & & $18: 26303160$ & $\mathrm{C}$ & A & 0.05 & HIST1H4A & 0.466 & $2.60 E-10$ \\
\hline rs57185274 & CACNA1A (intron 3) & & 19:13406972 & A & $\mathrm{T}$ & 0.05 & HIST1H4B & 1.034 & $2.04 E-10$ \\
\hline
\end{tabular}

a SNP in bold indicates the representative variant in each linkage disequilibrium block. SNP with superscript "\#” was associated with autism in a Chinese

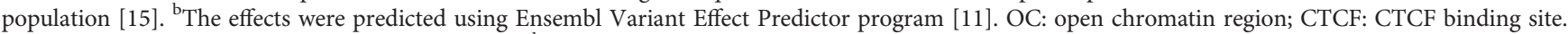
${ }^{\mathrm{c} C h r o m o s o m e: ~ b a s e ~ p a i r ~ p o s i t i o n ~ f r o m ~ G R C h 38 . p 2 . ~}{ }^{\mathrm{d}}$ A1: minor allele; A2: major allele. SNP: single-nucleotide polymorphism; MAF: minor allele frequency.

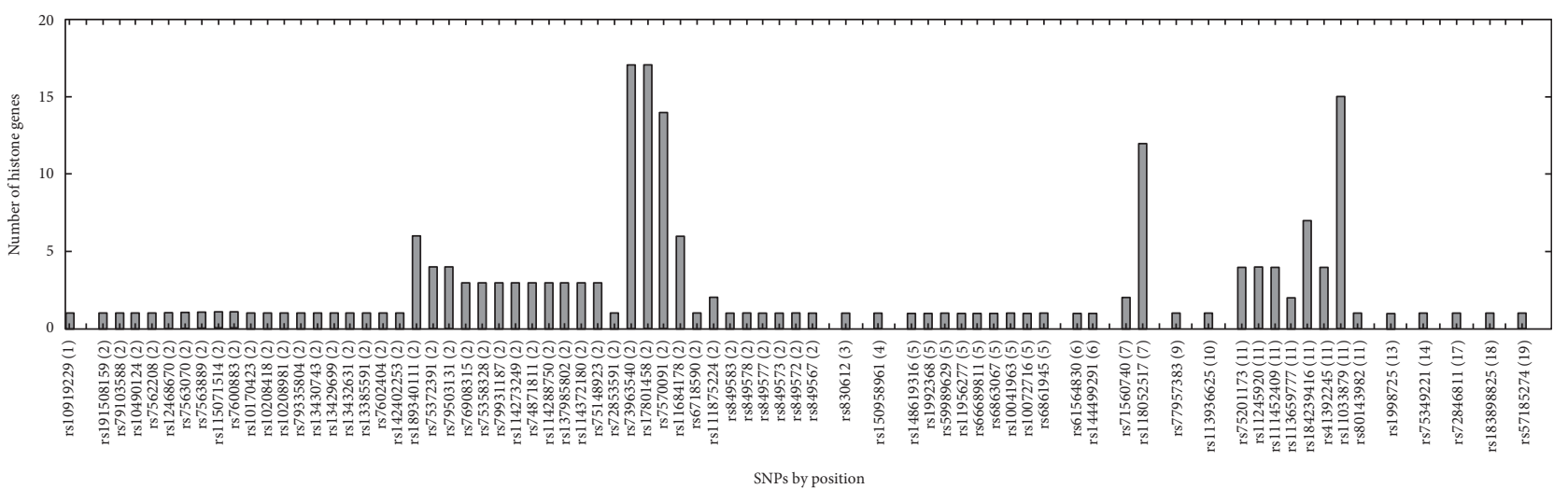

FIgURE 2: Number of histone genes with expression associated with SNPs. The figure in parentheses following the SNPs indicates the chromosomal number.

The identified eQTLs were further analyzed for their associations with the expression of nonhistone genes throughout the genome. The Bonferroni multiple testing based on $t$-statistic was also applied with a significance threshold value of $P=4.75 \times 10^{-6}$.

The functions of identified SNPs were examined using the Ensembl Variant Effect Predictor program [11] and RegulomeDB [12] (e.g., the motif of DNA footprinting assay, chromatin structure by DNA-seq, and protein binding by ChIP-seq).

\section{Results}

We observed numerous correlations amid the expression of the histone genes investigated in the current study (Figure 1). In particular, the expression of 29 genes showed correlations significantly $(P<0.05)$. Genome- wide association analysis showed that 74 SNPs were associated with the expression of the 29 histone genes $\left(P<2.97 \times 10^{-10}\right.$; Table 1$)$. Among them, 26 SNPs were simultaneously associated with the expression of multiple histone genes, and 5 out of 26 SNPs were associated with the expression of more than 10 histone genes (Figure 2). Thirty-one LD blocks were constructed covering the identified SNPs (Figure 3). The eQTLs corresponded to functional sites provided by various functional search sites. The rs79335804 had the most probable function with a RegulomeDB score of $2 b$ (Table S1 available online at https://doi.org/10.1155/ 2017/6202567).

Transcriptome-wide association analysis revealed 80 additional genes associated with the 31 identified eQTLs $\left(P<4.75 \times 10^{-6}\right.$; Table 2$)$. The genes encoding ribonuclease P RNA component H1 (RPPH1) and some small Cajal 

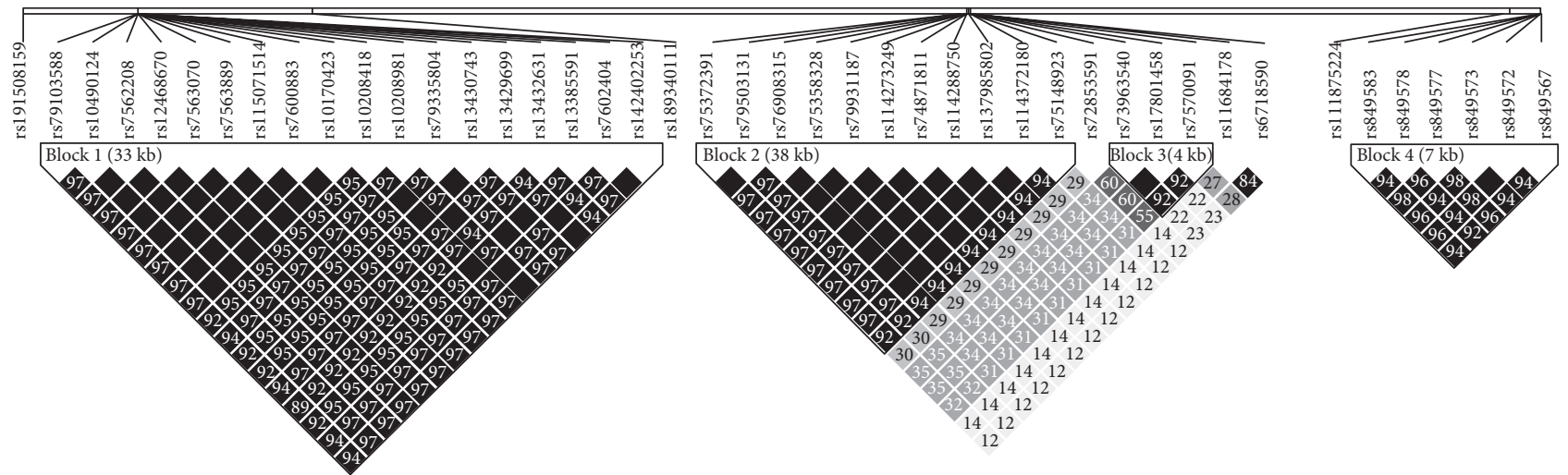

(a)

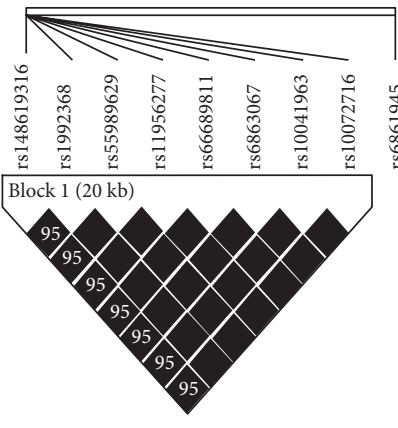

(b)

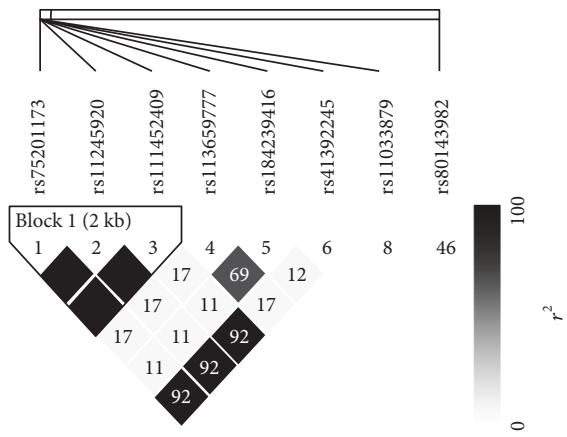

(c)

FIGURE 3: Linkage disequilibrium (LD) blocks of SNPs associated with the expression of histone genes. (a) 4 LD blocks in chromosome 2 (48891009-48924921, 141415941-141454893, 141710041-141714131, and 205737518-205744540), (b) 1 LD block in chromosome 5 (14080602-14101222), and (c) 1 LD block in chromosome 11 (1062030-1064929). No LD blocks were observed in chromosomes 6 and 7.

body-specific RNAs (scaRNAs), in particular, were associated with more than half of the eQTLs (>15 eQTLs; Table 2).

\section{Discussion}

We analyzed the eQTLs for simultaneously expressed histone genes. We found significant correlations amid the expression of 29 histone genes, which were all clustered in chromosome 1 or 6 . This clustered structure of the genes may serve to control simultaneous transcription, and this is supported by the observation that the expression of other histone genes not located on chromosome 1 or 6, including H1FX and H2A family members, was not correlated with those of the 29 selected genes. Furthermore, correlation estimates showed two subgroups nested within the large group (one with 21 genes and the other with 10 genes; with strong correlation coefficients of $r>0.7$ ), which likely provide a manageable unit for coordinating transcription.

The genome-wide eQTL analysis revealed that 12 loci were associated with the expression of multiple histone genes. The eQTLs were located on chromosomes 2, 7, and 11. Since 29 histone genes were all located on chromosome 1 or 6 , we suspect that the identified eQTLs were transacting. This suggests that many histone genes are simultaneously transcribed by remote regulators.

Functional analysis of the identified eQTLs suggests that they are very important for transcription. For example, rs79335804, an SNP within an eQTL on chromosome 2, was the binding motif for Kruppel-like factor 4 (Klf4) protein in various cells including LCLs. Klf4 was associated with chromosomal aberrations and can prevent cell proliferation by acting as a transcription factor [13]. The aberrant chromatin formation could be caused by overproduction of a histone dimer set (H2A-H2B or H3-H4) [14]. Thus, we suspect that there is an association between the chromosomal aberrations by Klf4 and histone gene mRNA expression. Rs 849578 within another eQTL on chromosome 2 was associated with autism in the Chinese Han population [15]. It is located in an intron of neuropilin 2 (NRP2) which may be an effector of apoptosis, proliferation, and neuronal development [16]. Histones are known to be related to developmental regulation [17], but additional study is required to elucidate underlying mechanism of the relationship between histones and NRP2.

Transcriptome-wide association analysis revealed that many nonhistone genes were also associated with the identified eQTLs. In particular, some genes were associated with 23 or more eQTLs. One was RPPH1, an RNA component of RNase P, which may assist in the cell cycle-dependent transcription of ribosomal RNAs (rRNAs) by associating with chromatin [18]. The expression of rRNAs increased from G1 to S and peaked at G2 [18]. The transcription of histone genes rapidly increased before the $S$ phase of the cell cycle and decreased shortly thereafter [1]. Thus, many eQLTs 
TABLE 2: Associations of the identified eQTLs with expression of histone and nonhistone genes all over the human genome.

\begin{tabular}{|c|c|c|c|c|c|c|c|c|}
\hline eQTL & Gene & $P$ & Gene & $P$ & Gene & $P$ & Gene & $P$ \\
\hline \multirow{4}{*}{ rs10919229 } & HIST1H4B (6) & $2.51 E-10$ & HIST1H3J (6) & $3.40 E-08$ & HIST1H3H (6) & $2.02 E-07$ & SASH3 (X) & $2.89 E-06$ \\
\hline & HIST1H2BM (6) & $4.38 E-09$ & HIST1H3B (6) & $4.64 E-08$ & HIST1H1E (6) & $2.50 E-07$ & HIST1H2AH (6) & $4.61 E-06$ \\
\hline & HIST1H1B (6) & $1.81 E-08$ & RPPH1 (14) & $5.82 E-08$ & HIST1H2AB (6) & $2.54 E-07$ & & \\
\hline & SCARNA13 (14) & $3.38 E-08$ & SCARNA12 (12) & $1.22 E-07$ & HIST1H2AM (6) & $9.76 E-07$ & & \\
\hline \multirow{5}{*}{ rs191508159 } & SNORA53 (12) & $4.38 E-13$ & SCARNA2 (1) & $5.28 E-08$ & HIST2H3C (1) & $1.41 E-07$ & HIST1H3 & $5.46 E-07$ \\
\hline & HIST1H4A (6) & $3.41 E-11$ & HIST1H1E (6) & $7.02 E-08$ & HIST1H2AL (6) & $1.99 E-07$ & G3BP2 (4) & $5.51 E-07$ \\
\hline & HIST1H2AJ (6) & $5.72 E-09$ & HIST2H2AB (1) & $7.35 E-08$ & HIST1H1B (6) & $2.67 E-07$ & HIST1H2AH (6) & $3.21 E-06$ \\
\hline & HIST1H3J (6) & $3.33 E-08$ & HIST1H2AB (6) & $1.02 E-07$ & HIST1H4K (6) & $2.99 E-07$ & HIST1H3A (6) & $4.06 E-06$ \\
\hline & HIST1H4J (6) & $4.63 E-08$ & HIST1H3B (6) & $1.14 E-07$ & HIST1H3I (6) & $5.12 E-07$ & HIST1H2BM (6) & $4.73 E-06$ \\
\hline \multirow{5}{*}{ rs13432631 } & HIST1H4B (6) & $1.78 E-11$ & HIST1H3B (6) & $1.13 E-08$ & HIST1H2AJ (6) & $1.86 E-07$ & HIST1H2AH (6) & $6.84 E-07$ \\
\hline & SCARNA12 (12) & $1.70 E-10$ & HIST1H2AB (6) & $2.83 E-08$ & HIST3H2BB (1) & $1.91 E-07$ & RPPH1 (14) & $7.69 E-07$ \\
\hline & HIST1H2BM (6) & $1.95 E-09$ & HIST1H3I (6) & $1.01 E-07$ & HIST1H3H (6) & $2.56 E-07$ & HIST1H3A (6) & $2.10 E-06$ \\
\hline & HIST1H1B (6) & $3.33 E-09$ & SCARNA13 (14) & $1.23 E-07$ & HIST1H4K (6) & $3.45 E-07$ & HIST1H4J (6) & $2.48 E-06$ \\
\hline & HIST1H3J (6) & $1.11 E-08$ & HIST1H1E (6) & $1.74 E-07$ & HIST2H2AB (1) & $6.28 E-07$ & HIST1H4A (6) & $2.81 E-06$ \\
\hline \multirow{7}{*}{ rs189340111 } & HIST1H4B (6) & $2.99 E-12$ & HIST1H3I (6) & $1.73 E-09$ & HIST1H4J (6) & $3.30 E-08$ & SCARNA & $4.27 E-07$ \\
\hline & HIST1H1F & $2.91 E-11$ & HIST2H 2 & $1.78 E-09$ & HIST1H & $3.60 E-08$ & PIG & $6.15 E-07$ \\
\hline & HIST1H1E & $6.90 E-11$ & HIST1H2AJ (6) & $2.58 E-09$ & HIST1H4 & $7.70 E-08$ & SCARNA & $1.55 E-06$ \\
\hline & HIST1H3J (6) & $8.17 E-11$ & HIST1H3H (6) & $3.93 E-09$ & HIST1H2AM (6) & $1.00 E-07$ & RPPH1 & $1.91 E-06$ \\
\hline & HIST1H2AH (6) & $2.34 E-10$ & HIST1H4 & $9.71 E-09$ & SCARNA1 & $1.05 E-07$ & HIST1H2 & $2.40 E-06$ \\
\hline & HIST1H2BM (6) & $2.58 E-10$ & HIST1H2AB (6) & $1.81 E-08$ & SCARNA7 (3) & $1.81 E-07$ & & \\
\hline & HIST1H3B (6) & $4.62 E-10$ & HIST3H2BB (1) & $3.14 E-08$ & HIST2H3C (1) & $2.90 E-07$ & & \\
\hline \multirow{7}{*}{ rs75372391 } & HIST1H2BM (6) & $6.19 E-12$ & HIST1H3I (6) & $5.56 E-09$ & HIST1H4A (6) & $3.57 E-08$ & HIST1H2 & $1.77 E-07$ \\
\hline & HIST1H4B (6) & $1.39 E-11$ & HIST1H2AJ (6) & $6.33 E-09$ & HIST1H2AH (6) & $5.13 E-08$ & HIST1H2AK (6) & $6.45 E-07$ \\
\hline & HIST1H1B (6) & $2.92 E-11$ & HIST1H2AB (6) & $7.88 E-09$ & HIST1H2AM (6) & $5.45 E-08$ & SCARNA7 (3) & $1.95 E-06$ \\
\hline & RPPH1 (14) & $1.53 E-10$ & HIST3H2BB (1) & $9.94 E-09$ & HIST1H4J (6) & $5.90 E-08$ & HIST1H3A (6) & $2.31 E-06$ \\
\hline & HIST1H3B (6) & $2.00 E-10$ & HIST1H3H (6) & $1.10 E-08$ & HIST2H3C (1) & $9.35 E-08$ & SCARNA2 (1) & $2.85 E-06$ \\
\hline & HIST1H1E (6) & $6.26 E-10$ & HIST2H2AB (1) & $2.33 E-08$ & SCARNA12 (12) & $9.42 E-08$ & & \\
\hline & HIST1H3J (6) & $1.15 E-09$ & HIST1H4K (6) & $3.21 E-08$ & HIST1H2AL (6) & $1.09 E-07$ & & \\
\hline \multirow{6}{*}{ rs72853591 } & HIST1H4B (6) & $9.68 E-11$ & SCARNA13 (14) & $1.03 E-08$ & SCARNA & $2.12 E-07$ & Н1S11Н & $2.69 E-06$ \\
\hline & HIST1H1B (6) & $5.88 E-10$ & HIST1H3J (6) & $1.17 E-08$ & HIST2H2AB (1) & $4.57 E-07$ & HIST1H2AL (6) & $2.72 E-06$ \\
\hline & HIST1H2BM (6) & $6.02 E-10$ & HIST1H1E (6) & $1.88 E-08$ & HIST1H4A (6) & $1.16 E-06$ & HIST1H4J (6) & $3.52 E-06$ \\
\hline & RPPH1 (14) & $1.96 E-09$ & HIST1H3H (6) & $1.56 E-07$ & HIST1H4K (6) & $1.57 E-06$ & HIST1H3A (6) & $4.33 E-06$ \\
\hline & HIST1H3B (6) & $4.11 E-09$ & HIST1H2AJ (6) & $1.74 E-07$ & SNORD17 (20) & $1.66 E-06$ & HIST2H3C (1) & $4.67 E-06$ \\
\hline & HIST1H3I (6) & $8.43 E-09$ & HIST1H2AB (6) & $1.84 E-07$ & SCARNA2 (1) & $2.24 E-06$ & & \\
\hline \multirow{9}{*}{ rs17801458 } & RPPH1 (14) & $1.31 E-15$ & HIST1H2AL (6) & $9.35 E-12$ & SCARNA2 (1) & $9.35 E-10$ & HIST1H2AK (6) & $1.75 E-06$ \\
\hline & HIST1H1B (6) & $1.62 E-14$ & HIST2H2AB (1) & $1.43 E-11$ & HIST1H3A (6) & $5.23 E-09$ & VGLL4 (3) & $1.88 E-06$ \\
\hline & HIST1H2BM (6) & $3.12 E-14$ & HIST2H3C (1) & $4.10 E-11$ & HIST1H2AG (6) & $6.96 E-09$ & SNORA53 (12) & $2.09 E-06$ \\
\hline & HIST1H3B (6) & $1.75 E-13$ & HIST1H4K (6) & $4.54 E-11$ & HIST1H2AH (6) & $1.34 E-08$ & WASF2 (1) & $3.49 E-06$ \\
\hline & HIST1H4B (6) & $4.90 E-13$ & HIST1H4J (6) & $4.67 E-11$ & SNORD17 (20) & $2.36 E-08$ & UBE2R2 (9) & $4.47 E-06$ \\
\hline & HIST1H2AB (6) & $8.94 E-13$ & HIST1H3H (6) & $5.05 E-11$ & SCARNA12 (12) & $2.60 E-08$ & & \\
\hline & HIST1H3J (6) & $1.28 E-12$ & HIST1H3I (6) & $6.66 E-11$ & SCARNA7 (3) & $1.91 E-07$ & & \\
\hline & HIST1H1E (6) & $3.33 E-12$ & HIST3H2BB (1) & $6.82 E-11$ & SCARNA13 (14) & $4.53 E-07$ & & \\
\hline & HIST1H2AJ (6) & $4.01 E-12$ & HIST1H4A (6) & $1.04 E-10$ & HIST1H2AM (6) & $1.16 E-06$ & & \\
\hline \multirow{4}{*}{ rs11684178 } & HIST1H2BM (6) & $6.71 E-12$ & HIST1H1E (6) & $3.59 E-10$ & HIST1H3A (6) & $2.78 E-09$ & NOL11 (17) & $4.61 E-07$ \\
\hline & RPPH1 (14) & $7.92 E-12$ & HIST1H4J (6) & $3.64 E-10$ & HIST2H3C (1) & $3.55 E-09$ & SCARNA12 (12) & $8.16 E-07$ \\
\hline & HIST1H1B (6) & $2.56 E-11$ & HIST1H4B (6) & $3.69 E-10$ & HIST3H2BB (1) & $9.84 E-09$ & SCARNA13 (14) & $1.09 E-06$ \\
\hline & HIST1H3B (6) & $6.72 E-11$ & HIST1H2AB (6) & $4.40 E-10$ & HIST1H4A (6) & $2.03 E-08$ & DDX17 (22) & $1.32 E-06$ \\
\hline
\end{tabular}


TABLe 2: Continued.

\begin{tabular}{|c|c|c|c|c|c|c|c|c|}
\hline eQTL & Gene & $P$ & Gene & $P$ & Gene & $P$ & Gene & $P$ \\
\hline & HIST2H2AB (1) & $1.04 E-10$ & IIST1H2AH (6) & $7.01 E-10$ & HIST1H4K (6) & $3.26 E-08$ & MRPS24 (7) & $3.22 E-06$ \\
\hline & HIST1H3J (6) & $1.10 E-10$ & HIST1H2AJ (6) & $8.58 E-10$ & SCARNA7 (3) & $.37 E-08$ & FASTKD2 (2) & $3.42 E-06$ \\
\hline & HIST1H2AL (6) & $1.67 E-10$ & HIST1H3H (6) & $1.56 E-09$ & HIST1H2AG (6) & $3.80 E-08$ & SNORD17 (20) & $3.99 E-06$ \\
\hline & HIST1H3I (6) & $3.41 E-10$ & SCARNA2 (1) & $2.47 E-09$ & HIST1H2AK (6) & $7.75 E-08$ & EFTUD2 (17) & $4.04 E-06$ \\
\hline \multirow{8}{*}{ rs6718590 } & HIST1H2BM (6) & $1.15 E-10$ & HIST1H1E (6) & $2.68 E-09$ & HIST1H3H (6) & $1.57 E-08$ & NOL1 & $4.12 E-07$ \\
\hline & RPPH1 (14) & $1.92 E-10$ & HIST1H3I (6) & $3.57 E-09$ & HIST1H3A (6) & $1.98 E-08$ & SCARNA12 (12) & $1.10 E-06$ \\
\hline & HIST1H1B (6) & $3.63 E-10$ & HIST1H4B (6) & $4.90 E-09$ & SCARNA2 (1) & $2.30 E-08$ & АРРВР2 (17) & $1.77 E-06$ \\
\hline & HIST1H2AL (6) & $4.03 E-10$ & HIST1H2AB (6) & $6.23 E-09$ & HIST1H2AG (6) & $8.36 E-08$ & ATP5A1 (18) & $3.68 E-06$ \\
\hline & HIST2H2AB (1) & $5.83 E-10$ & HIST1H2AH (6) & $6.89 E-09$ & HIST1H4A (6) & $1.92 E-07$ & PITPNB (22) & $3.92 E-06$ \\
\hline & HIST1H3J (6) & $9.78 E-10$ & HIST1H4J (6) & $9.48 E-09$ & SCARNA7 (3) & $2.51 E-07$ & & \\
\hline & HIST3H2BB (1) & $1.30 E-09$ & HIST2H3C (1) & $1.01 E-08$ & HIST1H4K (6) & $3.19 E-07$ & & \\
\hline & HIST1H3B (6) & $1.74 E-09$ & HIST1H2AJ (6) & $1.10 E-08$ & HIST1H2AK (6) & $3.22 E-07$ & & \\
\hline \multirow{7}{*}{ rs111875224 } & HIST1H2AM (6) & $1.24 E-11$ & SCARNA7 (3) & $2.79 E-08$ & HIST1H3H (6) & $2.63 E-07$ & GTF2 & $2.28 E-06$ \\
\hline & HIST1H & $8.51 E-11$ & HIST3H2BB (1) & $3.71 E-08$ & SCARNA2 (1) & $3.89 E-07$ & C18orf32 (18) & $2.68 E-06$ \\
\hline & HIST1H1B (6) & $7.63 E-10$ & HIST1H3J (6) & $9.07 E-08$ & HIST1H2AJ (6) & $4.51 E-07$ & CD63 (12) & $2.80 E-06$ \\
\hline & HIST1H1E (6) & $1.11 E-09$ & SCARNA12 (12) & $1.02 E-07$ & SCARN & $4.99 E-07$ & SERINC1 (6) & $3.24 E-06$ \\
\hline & HIST1H2BM (6) & $1.41 E-09$ & HIST1H3I (6) & $1.14 E-07$ & HIST1H3A (6) & $9.59 E-07$ & & \\
\hline & HIST1H3B (6) & $1.41 E-08$ & HIST1H2AB (6) & $1.32 E-07$ & HIST1H2AL (6) & $1.60 E-06$ & & \\
\hline & HIST1H2AH (6) & $2.27 E-08$ & HIST2H2AB (1) & $1.38 E-07$ & RPPH1 (14) & $1.87 E-06$ & & \\
\hline \multirow{6}{*}{ rs849577 } & HIST11 & $4.48 E-11$ & HIST11 & $3.42 E-08$ & HIST & $4.33 E-07$ & HIST & $2.03 E-06$ \\
\hline & SNOR & $3.16 E-10$ & HIST2H & $4.00 E-08$ & HIST & $5.65 E-07$ & HIST1H3H (6) & $2.06 E-06$ \\
\hline & HIST1H4J (6) & $8.79 E-10$ & HIST1H & $8.53 E-08$ & HIST & $6.70 E-07$ & RPPH1 (14) & $3.22 E-06$ \\
\hline & SCARNA2 (1) & $1.23 E-09$ & HIST1H2AL (6) & $1.35 E-07$ & HIST1H2A & $6.75 E-07$ & HIST1H & $4.42 E-06$ \\
\hline & HIST1H4K (6) & $2.46 E-09$ & HIST2H3C (1) & $2.09 E-07$ & SCARNA7 (3) & $1.71 E-06$ & & \\
\hline & HIST1H2AJ (6) & $1.12 E-08$ & HIST1H2AB (6) & $3.52 E-07$ & SNORD17 (20) & $1.77 E-06$ & & \\
\hline \multirow{4}{*}{ rs830612 } & HIST1H4B (6) & $1.28 E-10$ & HIST1H3B (6) & $1.16 E-08$ & SNORD17 (20) & $1.52 E-07$ & RPI & $6.10 E-07$ \\
\hline & HIST1H1B (6) & $6.19 E-10$ & HIST1H3H (6) & $1.67 E-08$ & SASH3 (X) & $1.74 E-07$ & HIST1H2AJ (6) & $6.67 E-07$ \\
\hline & HIST1H2BM (6) & $2.66 E-09$ & HIST1H1E (6) & $2.00 E-08$ & HIST1H2AH (6) & $2.54 E-07$ & SCARNA7 (3) & $1.56 E-06$ \\
\hline & HIST1H3J (6) & $1.05 E-08$ & HIST1H3I (6) & $4.17 E-08$ & HIST1H3A (6) & $3.80 E-07$ & HIST1H2AB (6) & $3.15 E-06$ \\
\hline \multirow{7}{*}{ rs150958961 } & HIST1H & $8.77 E-11$ & HIST1H1B (6) & $4.03 E-09$ & HIST1H & $3.84 E-08$ & HIST2H2 & $4.70 E-07$ \\
\hline & HIST1H3J (6) & $3.41 E-10$ & SCARI & $6.96 E-09$ & HIST1 & $4.81 E-08$ & SCAI & $5.49 E-07$ \\
\hline & HIST1H2AJ (6) & $3.78 E-10$ & HIST1H2AH (6) & $1.72 E-08$ & HIST1H3I (6) & $1.01 E-07$ & HIST1 & $1.04 E-06$ \\
\hline & HIST1H2AB (6) & $3.81 E-10$ & HIST1H2AL (6) & $1.86 E-08$ & HIST1H4B (6) & $1.38 E-07$ & SNORA57 (11) & $1.66 E-06$ \\
\hline & HIST1H3B (6) & $4.54 E-10$ & HIST1H2BM (6) & $2.30 E-08$ & HIST3H2BB (1) & $1.62 E-07$ & HIST1H2AG (6) & $2.46 E-06$ \\
\hline & RPPH1 (14) & $7.11 E-10$ & HIST1H4J (6) & $2.31 E-08$ & SNOR & $4.22 E-07$ & & \\
\hline & HIST1H1E (6) & $1.80 E-09$ & HIST2H3C (1) & $2.57 E-08$ & SNORD17 (20) & $4.60 E-07$ & & \\
\hline \multirow{7}{*}{ rs148619316 } & HIST1H4B (6) & $4.81 E-11$ & HIST1H3H (6) & $6.81 E-08$ & HIST1H2AJ (6) & $1.23 E-06$ & & $2.97 E-06$ \\
\hline & RPPH1 (14) & $3.50 E-09$ & HIST1H & $1.11 E-07$ & HIST & $1.33 E-06$ & THP & $3.14 E-06$ \\
\hline & HIST1H1B (6) & $4.92 E-09$ & HIST1H2AH (6) & $1.66 E-07$ & SNORD17 (20) & $1.52 E-06$ & SUPT5H (19) & $3.91 E-06$ \\
\hline & HIST1H3J (6) & $5.46 E-09$ & DDX28 (16) & $5.22 E-07$ & SCARNA12 (12) & $1.68 E-06$ & ZNF207 (17) & $4.48 E-06$ \\
\hline & HIST1H3B (6) & $9.95 E-09$ & HIST1H2AB (6) & $6.80 E-07$ & HIST1H2AK (6) & $1.69 E-06$ & & \\
\hline & HIST1H2BM (6) & $1.70 E-08$ & HIST2H2AB (1) & $1.14 E-06$ & C20orf11 (20) & $2.61 E-06$ & & \\
\hline & SASH3 (X) & $3.00 E-08$ & HIST3H2BB (1) & $1.22 E-06$ & SCARNA7 (3) & $2.67 E-06$ & & \\
\hline \multirow{4}{*}{ rs6861945 } & HIST1H3J (6) & $2.75 E-10$ & HIST1H4J (6) & $1.67 E-08$ & HIST1H3I (6) & $7.68 E-08$ & HIST1H2AK (6) & $1.18 E-06$ \\
\hline & HIST1H3B (6) & $4.30 E-09$ & HIST1H1E (6) & $2.30 E-08$ & HIST1H2AB (6) & $1.38 E-07$ & HIST2H2AB (1) & $1.32 E-06$ \\
\hline & HIST1H4B (6) & $4.44 E-09$ & HIST1H4K (6) & $3.40 E-08$ & SCARNA2 (1) & $1.55 E-07$ & SCARNA7 (3) & $2.25 E-06$ \\
\hline & HIST1H2BM (6) & $7.98 E-09$ & HIST1H2AH (6) & $3.78 E-08$ & HIST1H4A (6) & $1.55 E-07$ & SNORD17 (20) & $2.57 E-06$ \\
\hline
\end{tabular}


TABle 2: Continued.

\begin{tabular}{|c|c|c|c|c|c|c|c|c|}
\hline eQTL & Gene & $P$ & Gene & $P$ & Gene & $P$ & Gene & $P$ \\
\hline & HIST1H1B (6) & $1.04 E-08$ & HIST1H3H (6) & $4.52 E-08$ & SASH3 (X) & $4.33 E-07$ & HIST1H2AL (6) & $4.34 E-06$ \\
\hline & RPPH1 (14) & $1.11 E-08$ & HIST1H2AJ (6) & $5.09 E-08$ & HIST1H3A (6) & $8.63 E-07$ & & \\
\hline \multirow{6}{*}{ rs61564830 } & HIST1H3J (6) & $2.62 E-10$ & SCARNA2 (1) & $5.85 E-09$ & HIST1H3I (6) & $3.37 E-08$ & HIST1H2AB (6) & $2.54 E-06$ \\
\hline & HIST1H2AH (6) & $8.25 E-10$ & HIST1H1B (6) & $6.35 E-09$ & HIST1H2BM (6) & $3.45 E-08$ & HIST1H2AL (6) & $2.85 E-06$ \\
\hline & HIST1H1E (6) & $9.74 E-10$ & HIST1H2AJ (6) & $1.00 E-08$ & SCARNA13 (14) & $1.68 E-07$ & UBE2R2 (9) & $4.53 E-06$ \\
\hline & HIST1H3B (6) & $1.50 E-09$ & SNORD17 (20) & $1.23 E-08$ & RPPH1 (14) & $3.47 E-07$ & & \\
\hline & HIST1H3H (6) & $1.58 E-09$ & SCARNA7 (3) & $2.46 E-08$ & HIST2H2AB (1) & $2.23 E-06$ & & \\
\hline & HIST1H4B (6) & $2.05 E-09$ & HIST1H4A (6) & $2.75 E-08$ & MRPS31 (13) & $2.36 E-06$ & & \\
\hline \multirow{8}{*}{ rs144499291 } & HIST1H4A (6) & $2.88 E-10$ & HIST1H2AK (6) & $4.16 E-09$ & HIST1H4K (6) & $9.65 E-08$ & FAM122A (9) & $2.05 E-06$ \\
\hline & HIST1H1B (6) & $4.57 E-10$ & HIST1H3H (6) & $5.46 E-09$ & KPNB1 (17) & $1.48 E-07$ & RPPH1 (14) & $3.45 E-06$ \\
\hline & HIST1H2AJ (6) & $7.99 E-10$ & HIST1H3I (6) & $1.06 E-08$ & SNORD17 (20) & $1.49 E-07$ & HIST3H2BB (1) & $4.06 E-06$ \\
\hline & HIST1H1E (6) & $9.23 E-10$ & HIST1H2AB (6) & $1.12 E-08$ & HIST2H3C (1) & $2.10 E-07$ & & \\
\hline & HIST1H2BM (6) & $1.15 E-09$ & HIST2H2AB (1) & $3.99 E-08$ & HIST1H4J (6) & $2.73 E-07$ & & \\
\hline & HIST1H3J (6) & $2.45 E-09$ & HIST1H4B (6) & $4.58 E-08$ & SCARNA7 (3) & $2.83 E-07$ & & \\
\hline & HIST1H3B (6) & $2.85 E-09$ & SCARNA2 (1) & $5.61 E-08$ & HIST1H2AL (6) & $4.25 E-07$ & & \\
\hline & HIST1H2AH (6) & $4.05 E-09$ & SNORA53 (12) & $9.55 E-08$ & HIST1H3A (6) & $5.03 E-07$ & & \\
\hline \multirow{6}{*}{ rs71560740 } & HIST1H2AJ (6) & $1.48 E-10$ & HIST1H3I (6) & $6.75 E-09$ & RPPH1 (14) & $1.71 E-08$ & HIST1H2AH (6) & $4.57 E-07$ \\
\hline & HIST1H3A (6) & $2.41 E-10$ & HIST2H2AB (1) & $9.23 E-09$ & SNORA53 (12) & $2.32 E-08$ & HIST1H3H (6) & $9.55 E-07$ \\
\hline & HIST1H2AB (6) & $8.54 E-10$ & HIST1H4J (6) & $9.80 E-09$ & HIST1H3J (6) & $2.54 E-08$ & TDRD12 (19) & $3.26 E-06$ \\
\hline & HIST2H3C (1) & $1.32 E-09$ & HIST1H2BM (6) & $1.13 E-08$ & HIST1H1E (6) & $3.18 E-08$ & HIST1H2AM (6) & $3.74 E-06$ \\
\hline & HIST1H3B (6) & $3.46 E-09$ & HIST1H4A (6) & $1.27 E-08$ & HIST1H2AL (6) & $7.54 E-08$ & HIST1H4B (6) & $3.78 E-06$ \\
\hline & HIST1H1B (6) & $6.55 E-09$ & HIST1H4K (6) & $1.29 E-08$ & SCARNA2 (1) & $2.35 E-07$ & & \\
\hline \multirow{8}{*}{ rs118052517 } & HIST1H3J (6) & $8.16 E-13$ & HIST1H4K (6) & $2.14 E-10$ & HIST1H4B (6) & $2.72 E-09$ & HIST3H2BB (1) & $3.49 E-08$ \\
\hline & HIST1H4A (6) & $1.89 E-12$ & SCARNA2 (1) & $2.30 E-10$ & HIST1H2AH (6) & $2.81 E-09$ & LOXL4 (10) & $1.35 E-06$ \\
\hline & HIST1H2BM (6) & $2.40 E-12$ & HIST1H4J (6) & $2.62 E-10$ & HIST2H3C (1) & $6.12 E-09$ & KRT8 (12) & $1.53 E-06$ \\
\hline & HIST1H2AJ (6) & $3.64 E-12$ & HIST1H3I (6) & $2.77 E-10$ & SNORA53 (12) & $8.62 E-09$ & HIST1H2AM (6) & $1.63 E-06$ \\
\hline & HIST1H3B (6) & $6.16 E-12$ & HIST1H3H (6) & $2.95 E-10$ & HIST1H3A (6) & $1.04 E-08$ & TRNP1 (1) & $1.82 E-06$ \\
\hline & HIST1H1B (6) & $1.39 E-11$ & RPPH1 (14) & $1.53 E-09$ & HIST1H2AK (6) & $1.27 E-08$ & SCUBE2 (11) & $4.20 E-06$ \\
\hline & HIST1H1E (6) & $1.78 E-11$ & HIST1H2AL (6) & $1.73 E-09$ & SNORD17 (20) & $1.61 E-08$ & & \\
\hline & HIST1H2AB (6) & $1.16 E-10$ & HIST2H2AB (1) & $2.34 E-09$ & SCARNA7 (3) & $3.31 E-08$ & & \\
\hline \multirow{7}{*}{ rs77957383 } & HIST1H4B (6) & $1.85 E-10$ & HIST3H2BB (1) & $4.38 E-09$ & SCARNA12 (12) & $1.82 E-07$ & CDC5L (6) & $3.27 E-06$ \\
\hline & RPPH1 (14) & $3.19 E-10$ & HIST1H1E (6) & $2.17 E-08$ & SCARNA2 (1) & $2.84 E-07$ & HIST2H3C (1) & $4.43 E-06$ \\
\hline & HIST1H2BM (6) & $4.46 E-10$ & HIST2H2AB (1) & $2.20 E-08$ & HIST1H3H (6) & $7.97 E-07$ & & \\
\hline & HIST1H3B (6) & $1.04 E-09$ & HIST1H2AJ (6) & $2.95 E-08$ & HIST1H2AK (6) & $1.03 E-06$ & & \\
\hline & HIST1H1B (6) & $1.38 E-09$ & SCARNA7 (3) & $3.95 E-08$ & HIST1H4A (6) & $1.61 E-06$ & & \\
\hline & HIST1H3J (6) & $3.57 E-09$ & HIST1H3I (6) & $4.73 E-08$ & SCARNA13 (14) & $2.67 E-06$ & & \\
\hline & HIST1H2AB (6) & $3.98 E-09$ & HIST1H2AH (6) & $1.49 E-07$ & HIST1H2AL (6) & $3.22 E-06$ & & \\
\hline \multirow{5}{*}{ rs113936625 } & HIST1H4B (6) & $2.76 E-10$ & SCARNA13 (14) & $1.12 E-07$ & HIST1H3H (6) & $3.64 E-07$ & RPPH1 (14) & $2.97 E-06$ \\
\hline & HIST1H2BM (6) & $2.29 E-09$ & SCARNA12 (12) & $1.20 E-07$ & HIST1H2AJ (6) & $9.36 E-07$ & HIST1H2AL (6) & $3.00 E-06$ \\
\hline & HIST1H1B (6) & $8.82 E-09$ & HIST1H3B (6) & $1.36 E-07$ & HIST2H2AB (1) & $1.25 E-06$ & HIST1H3A (6) & $3.44 E-06$ \\
\hline & HIST1H2AM (6) & $1.42 E-08$ & HIST1H2AB (6) & $1.79 E-07$ & HIST1H3I (6) & $1.41 E-06$ & SNORD17 (20) & $3.63 E-06$ \\
\hline & HIST1H3J (6) & $3.32 E-08$ & HIST1H1E (6) & $2.11 E-07$ & HIST1H2AH (6) & $2.45 E-06$ & & \\
\hline \multirow{5}{*}{ rs75201173 } & HIST1H2BM (6) & $1.45 E-11$ & HIST1H2AK (6) & $2.05 E-09$ & STYX (14) & $4.33 E-07$ & HMHA1 (19) & $2.49 E-06$ \\
\hline & RPPH1 (14) & $6.79 E-11$ & HIST1H2AB (6) & $3.62 E-09$ & PRKAR1A (17) & $4.54 E-07$ & OSBPL11 (3) & $2.57 E-06$ \\
\hline & HIST2H3C (1) & $1.22 E-10$ & SCARNA2 (1) & $1.15 E-08$ & SMAD2 (18) & $5.85 E-07$ & FBXL12 (19) & $2.73 E-06$ \\
\hline & HIST1H1B (6) & $1.75 E-10$ & HIST1H2AL (6) & $1.56 E-08$ & EFTUD2 (17) & $6.59 E-07$ & FNBP1 (9) & $2.92 E-06$ \\
\hline & HIST1H3B (6) & $1.94 E-10$ & HIST2H2AB (1) & $3.33 E-08$ & ZFP91 (11) & $8.19 E-07$ & SLC44A2 (19) & $3.26 E-06$ \\
\hline
\end{tabular}


TABLe 2: Continued.

\begin{tabular}{|c|c|c|c|c|c|c|c|c|}
\hline eQTL & Gene & $P$ & Gene & $P$ & Gene & $P$ & Gene & $P$ \\
\hline & HIST1H4B (6) & $3.04 E-10$ & HIST1H3I (6) & $5.13 E-08$ & GBF1 (10) & $1.03 E-06$ & SCARNA12 (12) & $3.62 E-06$ \\
\hline & HIST1H3H (6) & $6.76 E-10$ & HIST1H2AH (6) & $5.81 E-08$ & АРВАЗ (19) & $1.15 E-06$ & SCARNA13 (14) & $4.24 E-06$ \\
\hline & HIST1H2AJ (6) & $6.95 E-10$ & HIST1H2AG (6) & $7.13 E-08$ & COX15 (10) & $1.90 E-06$ & ACLY (17) & $4.49 E-06$ \\
\hline & SCARNA7 (3) & $1.45 E-09$ & HIST1H4K (6) & $1.46 E-07$ & SERBP1 (1) & $2.00 E-06$ & & \\
\hline & HIST1H2AM (6) & $1.61 E-09$ & HIST3H2BB (1) & $1.64 E-07$ & HIST1H2BN (6) & $2.09 E-06$ & & \\
\hline & HIST1H1E (6) & $1.79 E-09$ & HIST1H4A (6) & $3.81 E-07$ & HIST1H3A (6) & $2.19 E-06$ & & \\
\hline & HIST1H3J (6) & $1.92 E-09$ & HIST1H4J (6) & $4.30 E-07$ & HIST1H2BO (6) & $2.22 E-06$ & & \\
\hline \multirow{9}{*}{ rs113659777 } & HIST1H2BM (6) & $2.89 E-11$ & HIST1H1E (6) & $1.72 E-09$ & SNORD17 (20) & $1.14 E-07$ & SCARNA12 (12) & $1.62 E-06$ \\
\hline & RPPH1 (14) & $7.27 E-11$ & HIST1H4K (6) & $3.31 E-09$ & HIST3H2BB (1) & $1.27 E-07$ & HTATSF1 (X) & $2.97 E-06$ \\
\hline & HIST1H4B (6) & $8.17 E-11$ & HIST1H2AJ (6) & $5.31 E-09$ & HIST1H2AK (6) & $1.40 E-07$ & AGGF1 (5) & $3.52 E-06$ \\
\hline & HIST1H1B (6) & $3.27 E-10$ & HIST1H2AB (6) & $7.80 E-09$ & SCARNA13 (14) & $2.84 E-07$ & MTFMT (15) & $3.89 E-06$ \\
\hline & HIST1H3B (6) & $6.97 E-10$ & HIST1H2AL (6) & $1.05 E-08$ & HIST1H4A (6) & $3.39 E-07$ & & \\
\hline & HIST2H2AB (1) & $9.75 E-10$ & SCARNA2 (1) & $1.72 E-08$ & HIST1H3I (6) & $3.63 E-07$ & & \\
\hline & HIST1H2AM (6) & $9.86 E-10$ & HIST1H4J (6) & $4.06 E-08$ & HIST1H2AG (6) & $4.70 E-07$ & & \\
\hline & HIST1H3J (6) & $1.08 E-09$ & HIST1H2AH (6) & $9.03 E-08$ & HIST1H3A (6) & $4.83 E-07$ & & \\
\hline & HIST1H3H (6) & $1.37 E-09$ & HIST2H3C (1) & $9.54 E-08$ & SCARNA7 (3) & $1.13 E-06$ & & \\
\hline \multirow{9}{*}{ rs184239416 } & HIST1H2BM (6) & $1.56 E-12$ & HIST2H2AB (1) & $2.30 E-09$ & SCARNA & $9.43 E-08$ & HIST2H3C (1) & $1.42 E-06$ \\
\hline & RPPH1 (14) & $2.76 E-12$ & HIST1H3H (6) & $2.98 E-09$ & HIST1H4 & $9.92 E-08$ & GNAI3 (1) & $1.60 E-06$ \\
\hline & HIST1H4B (6) & $9.13 E-12$ & HIST1H2AL (6) & $3.09 E-09$ & SCARNA13 (14) & $1.11 E-07$ & MAPRE1 (20) & $1.73 E-06$ \\
\hline & HIST1H1B (6) & $2.84 E-11$ & HIST1H2AJ (6) & $3.83 E-09$ & HIST1H & $1.46 E-07$ & RIOK3 (18) & $2.38 E-06$ \\
\hline & HIST1H3J (6) & $3.88 E-11$ & HIST1H2AM (6) & $8.62 E-09$ & CAPZA1 (1) & $1.84 E-07$ & HIST1H2AK (6) & $2.55 E-06$ \\
\hline & HIST1H3B (6) & $1.17 E-10$ & HIST1H2AB (6) & $1.50 E-08$ & HIST1H2AG (6) & $2.41 E-07$ & AGGF1 (5) & $2.75 E-06$ \\
\hline & HIST1H1E (6) & $2.06 E-10$ & SCARNA2 (1) & $3.83 E-08$ & HIST1H3A (6) & $2.62 E-07$ & ARL6IP6 (2) & $3.00 E-06$ \\
\hline & HIST3H2BB (1) & $2.37 E-10$ & HIST1H2AH (6) & $6.24 E-08$ & HIST1H4K (6) & $4.45 E-07$ & & \\
\hline & SNORD17 (20) & $1.19 E-09$ & SCARNA7 (3) & $6.81 E-08$ & HIST1H4J (6) & $4.76 E-07$ & & \\
\hline \multirow{11}{*}{ rs41392245 } & HIST1H2BM (6) & $4.19 E-11$ & HIST1H1E (6) & $3.18 E-09$ & HIST1H4K (6) & $2.14 E-07$ & HMHA1 (19) & $2.79 E-06$ \\
\hline & RPPH1 (14) & $1.07 E-10$ & HIST1H2AB (6) & $4.10 E-09$ & PRKAR1A (17) & $3.46 E-07$ & FBXL12 (19) & $3.05 E-06$ \\
\hline & HIST2H3C (1) & $1.34 E-10$ & HIST1H2AK (6) & $1.08 E-08$ & ZFP91 (11) & $3.49 E-07$ & SNORD17 (20) & $3.06 E-06$ \\
\hline & HIST1H3B (6) & $2.23 E-10$ & SCARNA2 (1) & $1.47 E-08$ & SCARNA12 (12) & $4.21 E-07$ & АРВA3 (19) & $3.07 E-06$ \\
\hline & HIST1H1B (6) & $2.90 E-10$ & HIST1H2AL (6) & $1.89 E-08$ & STYX (14) & $7.62 E-07$ & HIST1H2BN (6) & $3.26 E-06$ \\
\hline & HIST1H4B (6) & $3.59 E-10$ & HIST2H2AB (1) & $2.27 E-08$ & HIST1H4J (6) & $7.71 E-07$ & RDH5 (12) & $3.79 E-06$ \\
\hline & HIST1H3H (6) & $1.08 E-09$ & HIST1H2AG (6) & $2.99 E-08$ & HIST1H4A (6) & $9.00 E-07$ & ACLY (17) & $4.07 E-06$ \\
\hline & HIST1H2AJ (6) & $1.10 E-09$ & HIST1H2AH (6) & $4.46 E-08$ & HIST1H2BO (6) & $9.37 E-07$ & ZNF622 (5) & $4.27 E-06$ \\
\hline & SCARNA7 (3) & $1.16 E-09$ & HIST1H3I (6) & $7.59 E-08$ & SMAD2 (18) & $1.58 E-06$ & & \\
\hline & HIST1H2AM (6) & $2.39 E-09$ & HIST3H2BB (1) & $1.42 E-07$ & HIST1H3A (6) & $1.82 E-06$ & & \\
\hline & HIST1H3J (6) & $2.76 E-09$ & COX15 (10) & $1.96 E-07$ & EFTUD2 (17) & $2.57 E-06$ & & \\
\hline \multirow{9}{*}{ rs11033879 } & SNORA53 (12) & $2.29 E-13$ & HIST1H3J (6) & $2.08 E-11$ & HIST1H3A (6) & $4.97 E-10$ & HIST1H2 & $2.23 E-07$ \\
\hline & HIST1H2AJ (6) & $2.53 E-13$ & HIST1H4K (6) & $2.16 E-11$ & RPPH1 (14) & $1.18 E-09$ & OBFC2B (12) & $1.22 E-06$ \\
\hline & HIST1H1E (6) & $9.71 E-13$ & HIST1H2AB (6) & $3.14 E-11$ & HIST3H2BB (1) & $1.51 E-09$ & RUNDC1 (17) & $1.82 E-06$ \\
\hline & HIST1H3B (6) & $9.80 E-13$ & HIST1H4J (6) & $4.85 E-11$ & HIST1H4B (6) & $2.07 E-09$ & NHS (X) & $1.92 E-06$ \\
\hline & HIST2H2AB (1) & $1.12 E-12$ & HIST1H3I (6) & $7.46 E-11$ & HIST1H2AK (6) & $6.39 E-09$ & SVIL (10) & $4.02 E-06$ \\
\hline & HIST1H1B (6) & $1.18 E-12$ & SCARNA2 (1) & $1.10 E-10$ & HIST1H2AL (6) & $1.64 E-08$ & & \\
\hline & HIST1H4A (6) & $1.62 E-12$ & HIST1H3H (6) & $1.28 E-10$ & SCARNA7 (3) & $3.12 E-08$ & & \\
\hline & HIST1H2BM (6) & $4.53 E-12$ & HIST1H2AH (6) & $2.70 E-10$ & FN1 (2) & $3.67 E-08$ & & \\
\hline & HIST2H3C (1) & $1.15 E-11$ & SNORD17 (20) & $4.02 E-10$ & HIST1H2AM (6) & $1.68 E-07$ & & \\
\hline \multirow{2}{*}{ rs80143982 } & HIST1H4A & $2.65 E-10$ & NOTCH2NL (1) & $2.32 E-07$ & HIST1H3I (6) & $1.73 E-06$ & ZNF207 (17) & $4.57 E-06$ \\
\hline & HIST3H2BB (1) & $3.31 E-10$ & RNF26 (11) & $3.73 E-07$ & HIST1H2AJ (6) & $2.16 E-06$ & & \\
\hline
\end{tabular}


TABLE 2: Continued.

\begin{tabular}{|c|c|c|c|c|c|c|c|c|}
\hline eQTL & Gene & $P$ & Gene & $P$ & Gene & $P$ & Gene & $P$ \\
\hline & SNORA53 (12) & $2.42 E-08$ & SCARNA2 (1) & $6.10 E-07$ & SNORD17 (20) & $2.25 E-06$ & & \\
\hline & HIST1H1E (6) & $3.15 E-08$ & HIST2H3C (1) & $1.51 E-06$ & MRFAP1L1 (4) & $4.02 E-06$ & & \\
\hline \multirow{7}{*}{ rs75349221 } & HIST1H4A (6) & $2.75 E-10$ & HIST1H2BM (6) & $8.36 E-09$ & HIST1H3H (6) & $1.11 E-07$ & HIST1H2AB (6) & $1.91 E-06$ \\
\hline & HIST1H1E (6) & $4.32 E-10$ & HIST1H3I (6) & $8.42 E-09$ & SCARNA2 (1) & $1.45 E-07$ & NDUFA8 (9) & $2.23 E-06$ \\
\hline & HIST1H1B (6) & $7.02 E-10$ & SNORD17 (20) & $1.32 E-08$ & HIST1H4K (6) & $1.59 E-07$ & NDUFS1 (2) & $2.90 E-06$ \\
\hline & HIST1H3B (6) & $1.26 E-09$ & SNORA53 (12) & $1.96 E-08$ & HIST1H4J (6) & $2.64 E-07$ & RPPH1 (14) & $2.95 E-06$ \\
\hline & HIST1H2AJ (6) & $4.14 E-09$ & HIST1H4B (6) & $2.78 E-08$ & UBAP1 (9) & $3.35 E-07$ & TEX261 (2) & $3.35 E-06$ \\
\hline & SCARNA7 (3) & $7.10 E-09$ & HIST2H2AB (1) & $5.63 E-08$ & HIST2H3C (1) & $1.36 E-06$ & RUNDC1 (17) & $3.63 E-06$ \\
\hline & HIST1H3J (6) & $8.33 E-09$ & HIST1H2AH (6) & $8.54 E-08$ & SCARNA13 (14) & $1.61 E-06$ & HIST1H2AL (6) & $4.38 E-06$ \\
\hline \multirow{4}{*}{ rs72846811 } & HIST1H4A (6) & $1.90 E-11$ & HIST1H3I (6) & $1.06 E-07$ & SCARNA7 (3) & $3.49 E-07$ & HIST1H3A (6) & $2.08 E-06$ \\
\hline & SNORA53 (12) & $1.90 E-10$ & SCARNA2 (1) & $1.22 E-07$ & HIST1H3J (6) & $5.24 E-07$ & HIST1H2BM (6) & $2.77 E-06$ \\
\hline & HIST1H1E (6) & $8.56 E-09$ & HIST1H2AH (6) & $2.32 E-07$ & HIST2H3C (1) & $1.12 E-06$ & HIST2H2AB (1) & $2.79 E-06$ \\
\hline & HIST1H2AJ (6) & $7.92 E-08$ & HIST1H3B (6) & $2.44 E-07$ & HIST1H1B (6) & $1.24 E-06$ & & \\
\hline \multirow{5}{*}{ rs183898825 } & SNORA53 (12) & $9.65 E-11$ & HIST1H4J (6) & $2.52 E-08$ & HIST1H1E (6) & $2.74 E-07$ & HIST1H2AH (6) & $7.54 E-07$ \\
\hline & HIST1H4A (6) & $2.60 E-10$ & HIST1H2AJ (6) & $4.29 E-08$ & HIST2H2AB (1) & $4.28 E-07$ & SNORA70 (X) & $8.38 E-07$ \\
\hline & HIST1H4K (6) & $7.93 E-09$ & HIST2H3C (1) & $8.18 E-08$ & HIST1H3B (6) & $4.88 E-07$ & HIST1H1B (6) & $1.58 E-06$ \\
\hline & HIST1H3I (6) & $1.64 E-08$ & SCARNA7 (3) & $1.21 E-07$ & HIST1H3H (6) & $4.96 E-07$ & HIST1H2AB (6) & $2.66 E-06$ \\
\hline & SCARNA2 (1) & $1.69 E-08$ & SNORD17 (20) & $1.78 E-07$ & HIST1H3A (6) & $5.65 E-07$ & HIST1H3J (6) & $3.18 E-06$ \\
\hline \multirow{5}{*}{ rs57185274 } & HIST1H4B (6) & $2.04 E-10$ & HIST1H3J (6) & $4.54 E-08$ & HIST1H2AB (6) & $5.47 E-07$ & EPCAM (2) & $2.65 E-06$ \\
\hline & HIST1H2BM (6) & $3.32 E-09$ & HIST1H3B (6) & $7.51 E-08$ & HIST1H3H (6) & $6.20 E-07$ & HIST2H3C (1) & $2.79 E-06$ \\
\hline & HIST1H2AM (6) & $5.05 E-09$ & SCARNA12 (12) & $2.88 E-07$ & HIST1H2AJ (6) & $7.13 E-07$ & FNBP1 (9) & $3.03 E-06$ \\
\hline & HIST1H1B (6) & $7.66 E-09$ & SCARNA13 (14) & $4.61 E-07$ & HIST2H2AB (1) & $9.68 E-07$ & HIST3H2BB (1) & $3.24 E-06$ \\
\hline & HIST1H1E (6) & $2.02 E-08$ & HIST1H3I (6) & $5.12 E-07$ & HIST1H2AH (6) & $1.69 E-06$ & ZNF524 (19) & $4.57 E-06$ \\
\hline
\end{tabular}

Figure in parentheses indicates chromosome number.

identified in this study might be involved in the cell cycledependent expression of both RPPH1 and histone genes. Such a regulation of the eQTLs would be one of the key factors to solve their underlying mechanisms. The others identified with many eQTLs were the genes encoding scaRNAs (SCARNA2 and SCARNA7) located in the Cajal body, similar to the pre-mRNAs of histones, which move to the Cajal body for mRNA processing [3, 19].

Interestingly, many genes controlled by the same eQTLs as those for histones do not have polyadenylated structures [20]. In particular, the genes associated with more than 10 eQTLs were all nonpolyadenylated. They were snoRNAs, scaRNAs, and RPPH1. Considering that histones are also nonpolyadenylated, this may help us to understand the transcriptional regulation of histone genes by these eQTLs.

The expressions of histone genes play an important role in controlling chromatin accessibility [21]. Improper expression of histone genes has been associated with tumorigenesis [22-24]. Expression of NPAT, a transcriptional activator for histone genes, is also associated with human tumorigenesis [25]. The influence of histone genes on tumor developments might be supported by the eQTLs identified in the current study, because some of the eQTLs were located within anticipated tumor suppressor genes such as low-density lipoprotein receptor-related protein (LRP1B) and utrophin (UTRN) $[26,27]$.
In conclusion, we identified 31 eQTLs for histone genes. The eQTLs were also associated with nonhistone genes that exhibited both a cell cycle-dependent expression and a nonpolyadenylated RNA structure. Further investigations are required to understand the mechanisms regulating the transcription of the histone and nonhistone genes identified in this study and to appreciate their influence on cancer and other diseases. Moreover, identification of eQTLs using disease-specific cell types would provide resolute mechanisms by diseases.

\section{Conflicts of Interest}

The authors declare that they have no competing interests.

\section{Acknowledgments}

This work was funded by the National Research Foundation of Korea, the Ministry of Education, Science, and Technology (Grant no. NRF-2012M3A9D1054705).

\section{References}

[1] W. F. Marzluff and R. J. Duronio, "Histone mRNA expression: multiple levels of cell cycle regulation and important developmental consequences," Current Opinion in Cell Biology, vol. 14, no. 6, pp. 692-699, 2002. 
[2] G. Li, X. Ruan, R. K. Auerbach et al., "Extensive promotercentered chromatin interactions provide a topological basis for transcription regulation," Cell, vol. 148, no. 1-2, pp. 8498, 2012.

[3] L. S. Shopland, M. Byron, J. L. Stein, J. B. Lian, G. S. Stein, and J. B. Lawrence, "Replication-dependent histone gene expression is related to Cajal body (CB) association but does not require sustained CB contact," Molecular Biology of the Cell, vol. 12, no. 3, pp. 565-576, 2001.

[4] G. S. Stein, A. J. van Wijnen, J. L. Stein et al., "An architectural perspective of cell-cycle control at the G1/S phase cell-cycle transition," Journal of Cellular Physiology, vol. 209, no. 3, pp. 706-710, 2006.

[5] Genomes Project Consortium, G. R. Abecasis, A. Auton et al., "An integrated map of genetic variation from 1,092 human genomes," Nature, vol. 491, no. 7422, pp. 56-65, 2012.

[6] T. Lappalainen, M. Sammeth, M. R. Friedlander et al., "Transcriptome and genome sequencing uncovers functional variation in humans," Nature, vol. 501, no. 7468, pp. 506-511, 2013.

[7] P. A. t Hoen, M. R. Friedlander, J. Almlof et al., "Reproducibility of high-throughput mRNA and small RNA sequencing across laboratories," Nature Biotechnology, vol. 31, no. 11, pp. 1015-1022, 2013.

[8] S. Purcell, B. Neale, K. Todd-Brown et al., "Plink: a tool set for whole-genome association and population-based linkage analyses," American Journal of Human Genetics, vol. 81, no. 3, pp. 559-575, 2007.

[9] J. C. Barrett, B. Fry, J. Maller, and M. J. Daly, "Haploview: analysis and visualization of LD and haplotype maps," Bioinformatics, vol. 21, no. 2, pp. 263-265, 2005.

[10] S. B. Gabriel, S. F. Schaffner, H. Nguyen et al., "The structure of haplotype blocks in the human genome," Science, vol. 296, no. 5576, pp. 2225-2229, 2002.

[11] W. McLaren, B. Pritchard, D. Rios, Y. Chen, P. Flicek, and F. Cunningham, "Deriving the consequences of genomic variants with the Ensembl API and SNP Effect Predictor," Bioinformatics, vol. 26, no. 16, pp. 2069-2070, 2010.

[12] A. P. Boyle, E. L. Hong, M. Hariharan et al., "Annotation of functional variation in personal genomes using RegulomeDB," Genome Research, vol. 22, no. 9, pp. 1790-1797, 2012.

[13] E. A. El-Karim, E. G. Hagos, A. M. Ghaleb, B. Yu, and V. W. Yang, "Kruppel-like factor 4 regulates genetic stability in mouse embryonic fibroblasts," Molecular Cancer, vol. 12, p. 89, 2013.

[14] D. Meeks-Wagner and L. H. Hartwell, "Normal stoichiometry of histone dimer sets is necessary for high fidelity of mitotic chromosome transmission," Cell, vol. 44, no. 1, pp. 43-52, 1986.

[15] S. Wu, W. Yue, M. Jia et al., "Association of the neuropilin-2 (NRP2) gene polymorphisms with autism in Chinese Han population," American Journal of Medical Genetics Part B: Neuropsychiatric Genetics, vol. 144B, no. 4, pp. 492-495, 2007.

[16] C. Chen, M. Li, H. Chai, H. Yang, W. E. Fisher, and Q. Yao, "Roles of neuropilins in neuronal development, angiogenesis, and cancers," World Journal of Surgery, vol. 29, no. 3, pp. 271-275, 2005.

[17] R. W. Old and H. R. Woodland, "Histone genes: not so simple after all," Cell, vol. 38, no. 3, pp. 624-626, 1984.

[18] R. Reiner, N. Krasnov-Yoeli, Y. Dehtiar, and N. Jarrous, "Function and assembly of a chromatin-associated RNase P that is required for efficient transcription by RNA polymerase I," PLoS One, vol. 3, no. 12, article e4072, 2008.
[19] X. Darzacq, B. E. Jady, C. Verheggen, A. M. Kiss, E. Bertrand, and T. Kiss, "Cajal body-specific small nuclear RNAs: a novel class of 2'-O-methylation and pseudouridylation guide RNAs," The EMBO Journal, vol. 21, no. 11, pp. 2746-2756, 2002.

[20] L. Yang, M. O. Duff, B. R. Graveley, G. G. Carmichael, and L. L. Chen, "Genomewide characterization of non-polyadenylated RNAs," Genome Biology, vol. 12, no. 2, article R16, 2011.

[21] S. Misri, S. Pandita, R. Kumar, and T. K. Pandita, "Telomeres, histone code, and DNA damage response," Cytogenetic and Genome Research, vol. 122, no. 3-4, pp. 297-307, 2008.

[22] K. Yoshioka, Y. Atsumi, H. Nakagama, and H. Teraoka, "Development of cancer-initiating cells and immortalized cells with genomic instability," World Journal of Stem Cells, vol. 7, no. 2, pp. 483-489, 2015.

[23] M. Piscopo, G. Campisi, G. Colella et al., "H3 and H3.3 histone mRNA amounts and ratio in oral squamous cell carcinoma and leukoplakia," Oral Diseases, vol. 12, no. 2, pp. 130-136, 2006.

[24] T. Iwaya, T. Fukagawa, Y. Suzuki et al., "Contrasting expression patterns of histone mRNA and microRNA 760 in patients with gastric cancer," Clinical Cancer Research, vol. 19, no. 23, pp. 6438-6449, 2013.

[25] G. Gao, A. P. Bracken, K. Burkard et al., "NPAT expression is regulated by E2F and is essential for cell cycle progression," Molecular and Cellular Biology, vol. 23, no. 8, pp. 2821-2833, 2003.

[26] C. X. Liu, Y. Li, L. M. Obermoeller-McCormick, A. L. Schwartz, and G. Bu, "The putative tumor suppressor LRP1B, a novel member of the low density lipoprotein (LDL) receptor family, exhibits both overlapping and distinct properties with the LDL receptor-related protein," The Journal of Biological Chemistry, vol. 276, no. 31, pp. 28889-28896, 2001.

[27] Y. Li, J. Huang, Y. L. Zhao et al., "UTRN on chromosome 6q24 is mutated in multiple tumors," Oncogene, vol. 26, no. 42, pp. 6220-6228, 2007. 

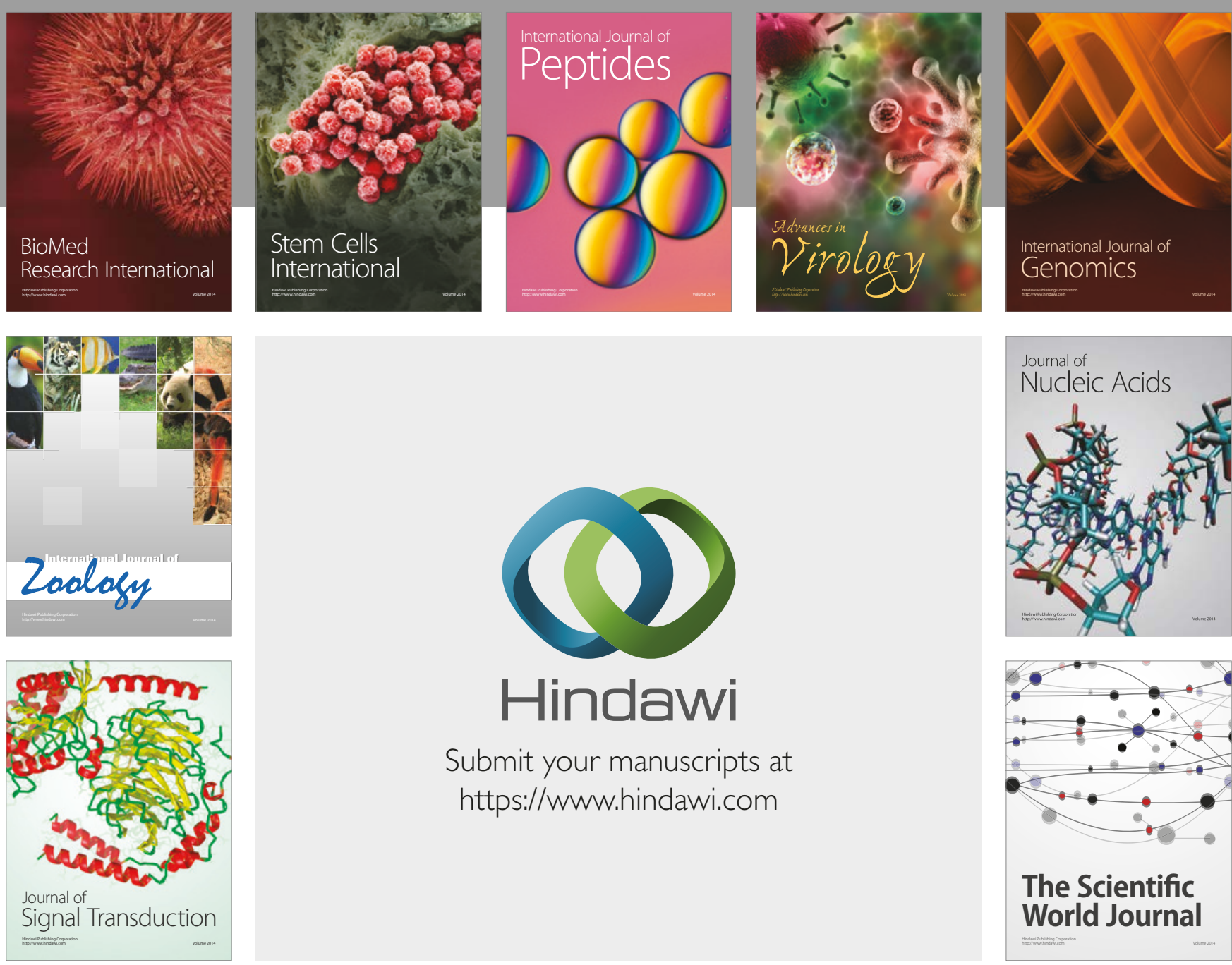

Submit your manuscripts at

https://www.hindawi.com
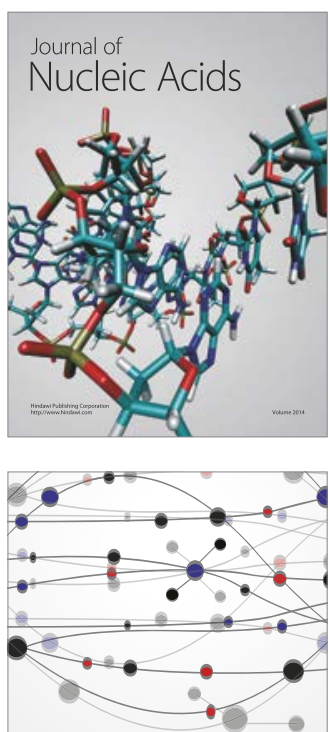

The Scientific World Journal

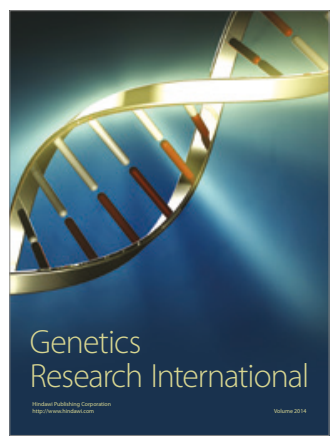

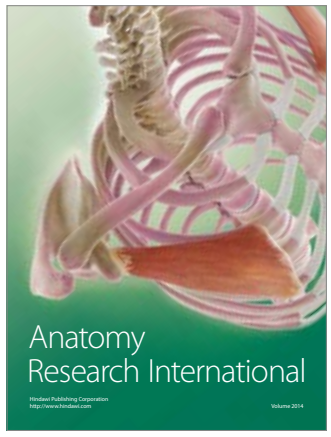

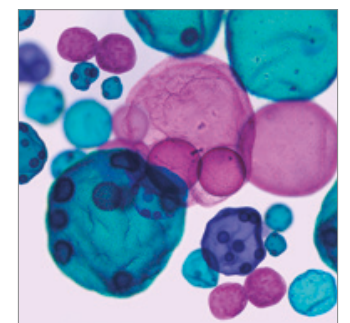

International Journal of Microbiology
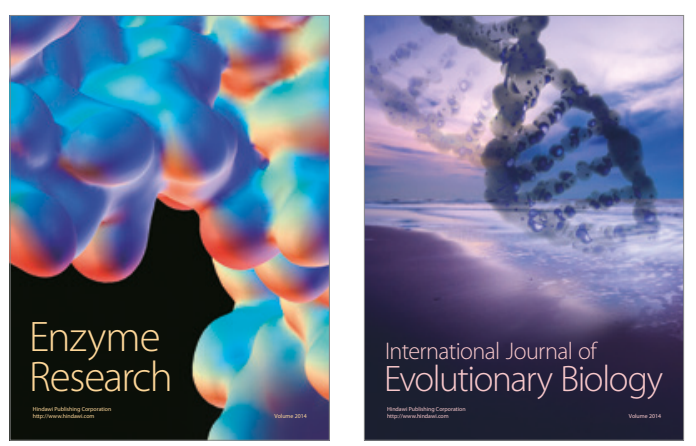
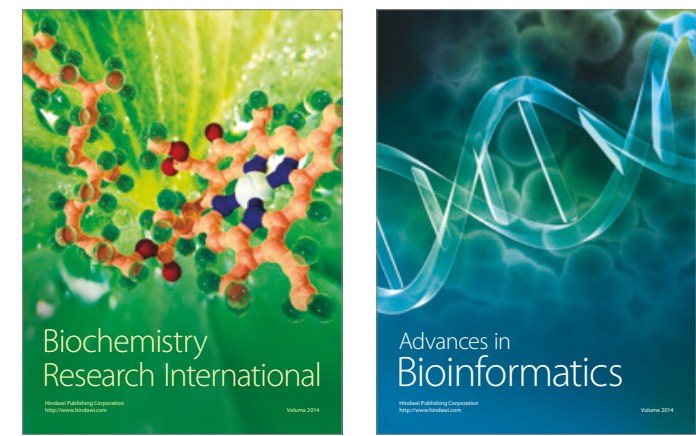

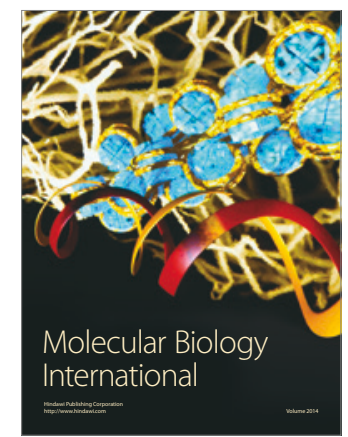

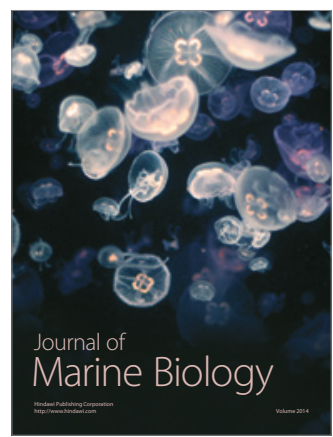

